Louisiana State University

LSU Digital Commons

Faculty Publications

Department of Physics \& Astronomy

$1-1-2019$

\title{
OGLE-2018-BLG-1011Lb,c: Microlensing planetary system with two giant planets orbiting a low-mass star
}

\author{
Cheongho Han \\ Chungbuk National University \\ David P. Bennett \\ NASA Goddard Space Flight Center \\ Andrzej Udalski \\ Uniwersytet Warszawski \\ Andrew Gould \\ Korea Astronomy \\ Ian A. Bond \\ Massey University Auckland
}

See next page for additional authors

Follow this and additional works at: https://digitalcommons.Isu.edu/physics_astronomy_pubs

\section{Recommended Citation}

Han, C., Bennett, D., Udalski, A., Gould, A., Bond, I., Shvartzvald, Y., Nikolaus, K., Hundertmark, M., Bozza, V., Cassan, A., Hirao, Y., Bachelet, E., Fouqué, P., Albrow, M., Chung, S., Hong, K., Hwang, K., Lee, C., Ryu, Y., Shin, I., Yee, J., Jung, Y., Cha, S., Kim, D., Kim, D., Kim, H., Kim, S., Lee, D., Lee, Y., Park, B., Pogge, R., Mróz, P., \& Szymański, M. (2019). OGLE-2018-BLG-1011Lb,c: Microlensing planetary system with two giant planets orbiting a low-mass star. Astronomical Journal, 158 (3) https://doi.org/10.3847/1538-3881/ab2f74

This Article is brought to you for free and open access by the Department of Physics \& Astronomy at LSU Digital Commons. It has been accepted for inclusion in Faculty Publications by an authorized administrator of LSU Digital Commons. For more information, please contact ir@lsu.edu. 


\section{Authors}

Cheongho Han, David P. Bennett, Andrzej Udalski, Andrew Gould, lan A. Bond, Yossi Shvartzvald, Kay Sebastian Nikolaus, Markus Hundertmark, Valerio Bozza, Arnaud Cassan, Yuki Hirao, Etienne Bachelet, Pascal Fouqué, Michael D. Albrow, Sun Ju Chung, Kyeongsoo Hong, Kyu Ha Hwang, Chung Uk Lee, Yoon Hyun Ryu, In Gu Shin, Jennifer C. Yee, Youn Kil Jung, Sang Mok Cha, Doeon Kim, Dong Jin Kim, Hyoun Woo Kim, Seung Lee Kim, Dong Joo Lee, Yongseok Lee, Byeong Gon Park, Richard W. Pogge, Przemek Mróz, and Michał K. Szymański 


\title{
OGLE-2018-BLG-1011Lb,c: Microlensing Planetary System with Two Giant Planets Orbiting a Low-mass Star
}

\author{
Cheongho Han ${ }^{1}$ (i), David P. Bennett ${ }^{2,3,38}$ (10), Andrzej Udalski ${ }^{4,39}$, Andrew Gould ${ }^{5,6,7,40}$, Ian A. Bond ${ }^{8,38}$, \\ Yossi Shvartzvald ${ }^{9,40,41}$ (1) , Kay-Sebastian Nikolaus ${ }^{10}$, Markus Hundertmark ${ }^{10}$, Valerio Bozza ${ }^{11,12}$ (10), Arnaud Cassan ${ }^{13}$, \\ Yuki Hirao ${ }^{14,38}$, Etienne Bachelet ${ }^{15}$, Pascal Fouqué ${ }^{16,17}$ \\ (Leading Authors), \\ Michael D. Albrow ${ }^{18}$, Sun-Ju Chung ${ }^{5,19}$ (1), Kyeongsoo Hong ${ }^{1}$, Kyu-Ha Hwang ${ }^{5}$ (1) , Chung-Uk Lee $^{5}$, Yoon-Hyun Ryu ${ }^{5}$ (1), \\ In-Gu Shin ${ }^{5}$, Jennifer C. Yee ${ }^{20}$, Youn Kil Jung ${ }^{5}$, Sang-Mok Cha ${ }^{5,21}$, Doeon Kim ${ }^{1}$, Dong-Jin Kim ${ }^{5}$, Hyoun-Woo Kim ${ }^{5}$, \\ Seung-Lee Kim ${ }^{5,19}$, Dong-Joo Lee ${ }^{5}$, Yongseok Lee ${ }^{5,21}$, Byeong-Gon Park ${ }^{5,19}$, Richard W. Pogge ${ }^{6}$ \\ (The KMTNet Collaboration), \\ Przemek Mróz ${ }^{4}$, Michał K. Szymański ${ }^{4}$, Jan Skowron ${ }^{4}$ (D), Radek Poleski ${ }^{6}$, Igor Soszyński ${ }^{4}$, Paweł Pietrukowicz ${ }^{4}$ (1), \\ Szymon Kozłowski ${ }^{4}$, Krzysztof Ulaczyk ${ }^{22}$ (D), Krzysztof A. Rybicki ${ }^{4}$, Patryk Iwanek ${ }^{4}$ (i), Marcin Wrona ${ }^{4}$ \\ (The OGLE Collaboration),
}

Fumio $\mathrm{Abe}^{23}$, Richard Barry ${ }^{2}$, Aparna Bhattacharya ${ }^{2,3}$, Martin Donachie ${ }^{24}$, Akihiko Fukui ${ }^{25}$ (1) , Yoshitaka Itow $^{23}$ (1), Kohei Kawasaki $^{14}$, Iona Kondo ${ }^{14}$, Naoki Koshimoto ${ }^{26,27}$ (D), Man Cheung Alex Li ${ }^{24}$, Yutaka Matsubara ${ }^{23}$, Yasushi Muraki ${ }^{23}$, Shota Miyazaki $^{14}$ (i), Masayuki Nagakane ${ }^{14}$, Clément Ranc ${ }^{2}$, Nicholas J. Rattenbury ${ }^{24}$ (1), Haruno Suematsu ${ }^{14}$, Denis J. Sullivan ${ }^{28}$, Takahiro Sumi ${ }^{14}$, Daisuke Suzuki ${ }^{29}$ (i), Paul J. Tristram ${ }^{30}$, Atsunori Yonehara ${ }^{31}$

(The MOA Collaboration),
Shude $\mathrm{Mao}^{32,33,34}$, Tianshu Wang ${ }^{32}$, Weicheng Zang ${ }^{32}$, Wei Zhu ${ }^{35}$, Matthew T. Penny ${ }^{6,41}$ (i)

(The CFHT Collaboration),

and

Charles A. Beichman ${ }^{9}$, Geoffery Bryden ${ }^{36}$, Sebastiano Calchi Novati ${ }^{9}$, B. Scott Gaudi ${ }^{6}$ (1) Calen B. Henderson $^{9}$ (), Savannah Jacklin ${ }^{37}$ (1), and Keivan G. Stassun ${ }^{37}$ (10

(The UKIRT Microlensing Team)

\footnotetext{
${ }^{1}$ Department of Physics, Chungbuk National University, Cheongju 28644, Republic of Korea; cheongho@astroph.chungbuk.ac.kr ${ }^{2}$ Code 667, NASA Goddard Space Flight Center, Greenbelt, MD 20771, USA ${ }^{3}$ Department of Astronomy, University of Maryland, College Park, MD 20742, USA

${ }_{5}^{4}$ Warsaw University Observatory, Al. Ujazdowskie 4, 00-478 Warszawa, Poland

${ }^{5}$ Korea Astronomy and Space Science Institute, Daejon 34055, Republic of Korea

${ }^{6}$ Department of Astronomy, Ohio State University, 140 W. 18th Avenue, Columbus, OH 43210, USA

${ }^{7}$ Max Planck Institute for Astronomy, Königstuhl 17, D-69117 Heidelberg, Germany

${ }^{8}$ Institute of Natural and Mathematical Sciences, Massey University, Auckland 0745, New Zealand

${ }^{9}$ IPAC, Mail Code 100-22, Caltech, 1200 E. California Boulevard, Pasadena, CA 91125, USA

${ }^{10}$ Zentrum für Astronomie der Universität Heidelberg, Astronomisches Rechen-Institut, Mönchhofstr. 12-14, D-69120 Heidelberg, Germany

${ }^{11}$ Dipartimento di Fisica "E. R. Caianiello," Université di Salerno, Via Giovanni Paolo II, I-84084 Fisciano (SA), Italy

12 Istituto Nazionale di Fisica Nucleare, Sezione di Napoli, Via Cintia, I-80126 Napoli, Italy

${ }^{13}$ Institut d'Astrophysique de Paris, Sorbonne Université, CNRS, UMR 7095, 98 bis bd Arago, F-75014 Paris, France

${ }^{14}$ Department of Earth and Space Science, Graduate School of Science, Osaka University, Toyonaka, Osaka 560-0043, Japan

${ }^{15}$ Las Cumbres Observatory, 6740 Cortona Drive, Suite 102, Goleta, CA 93117, USA

${ }^{16}$ CFHT Corporation, 65-1238 Mamalahoa Hwy, Kamuela, Hawaii 96743, USA ${ }^{17}$ Université de Toulouse, UPS-OMP, IRAP, Toulouse, France

${ }^{18}$ University of Canterbury, Department of Physics and Astronomy, Private Bag 4800, Christchurch 8020, New Zealand

${ }^{19}$ University of Science and Technology (UST), 217 Gajeong-ro Yuseong-gu, Daejeon 34113, Republic of Korea

${ }^{20}$ Center for Astrophysics, Harvard \& Smithsonian, 60 Garden Street, Cambridge, MA 02138, USA

${ }^{21}$ School of Space Research, Kyung Hee University, Yongin, Kyeonggi 17104, Republic of Korea

${ }^{22}$ Department of Physics, University of Warwick, Gibbet Hill Road, Coventry, CV4 7AL, UK

${ }^{23}$ Institute for Space-Earth Environmental Research, Nagoya University, Nagoya 464-8601, Japan

${ }^{24}$ Department of Physics, University of Auckland, Private Bag 92019, Auckland, New Zealand

${ }^{25}$ Okayama Astrophysical Observatory, National Astronomical Observatory of Japan, 3037-5 Honjo, Kamogata, Asakuchi, Okayama 719-0232, Japan

${ }^{26}$ Department of Astronomy, Graduate School of Science, The University of Tokyo, 7-3-1 Hongo, Bunkyo-ku, Tokyo 113-0033, Japan

27 National Astronomical Observatory of Japan, 2-21-1 Osawa, Mitaka, Tokyo 181-8588, Japan

${ }^{28}$ School of Chemical and Physical Sciences, Victoria University, Wellington, New Zealand

${ }^{29}$ Institute of Space and Astronautical Science, Japan Aerospace Exploration Agency, 3-1-1 Yoshinodai, Chuo, Sagamihara, Kanagawa, 252-5210, Japan

${ }^{30}$ University of Canterbury Mt. John Observatory, P.O. Box 56, Lake Tekapo 8770, New Zealand

${ }^{31}$ Department of Physics, Faculty of Science, Kyoto Sangyo University, 603-8555 Kyoto, Japan

${ }^{32}$ Physics Department and Tsinghua Centre for Astrophysics, Tsinghua University, Beijing 100084, People's Republic of China

${ }^{33}$ National Astronomical Observatories, Chinese Academy of Sciences, A20 Datun Road, Chaoyang District, Beijing 100012, People's Republic of China

${ }^{34}$ Jodrell Bank Centre for Astrophysics, Alan Turing Building, University of Manchester, Manchester M13 9PL, UK

${ }^{35}$ Canadian Institute for Theoretical Astrophysics, University of Toronto, 60 St. George Street, Toronto, ON M5S 3H8, Canada

${ }^{36}$ Jet Propulsion Laboratory, California Institute of Technology, 4800 Oak Grove Drive, Pasadena, CA 91109, USA
} 


\footnotetext{
${ }^{37}$ Vanderbilt University, Department of Physics \& Astronomy, Nashville, TN 37235, USA Received 2019 April 10; revised 2019 June 25; accepted 2019 July 2; published 2019 August 19
}

\begin{abstract}
We report a multiplanetary system found from the analysis of microlensing event OGLE-2018-BLG-1011, for which the light curve exhibits a double-bump anomaly around the peak. We find that the anomaly cannot be fully explained by the binary-lens or binary-source interpretations and its description requires the introduction of an additional lens component. The 3L1S (three lens components and a single source) modeling yields three sets of solutions, in which one set of solutions indicates that the lens is a planetary system in a binary, while the other two sets imply that the lens is a multiplanetary system. By investigating the fits of the individual models to the detailed light curve structure, we find that the multiple-planet solution with planet-to-host mass ratios $\sim 9.5 \times 10^{-3}$ and $\sim 15 \times 10^{-3}$ are favored over the other solutions. From the Bayesian analysis, we find that the lens is composed of two planets with masses $1.8_{-1.1}^{+3}{ }^{4} M_{\mathrm{J}}$ and $2.8_{-1.7}^{+5.1} M_{\mathrm{J}}$ around a host with a mass $0.18_{-0.10}^{+0.33} M_{\odot}$ and located at a distance $7.1_{-1.5}^{+1.1} \mathrm{kpc}$. The estimated distance indicates that the lens is the farthest system among the known multiplanetary systems. The projected planet-host separations are $a_{\perp, 2}=1.8_{-1.5}^{+2.1}$ au $\left(0.8_{-0.6}^{+0.9}\right.$ au) and $a_{\perp, 3}=0.8_{-0.6}^{+0.9}$ au, where the values of $a_{\perp, 2}$ inside and outside the parenthesis are the separations corresponding to the two degenerate solutions, indicating that both planets are located beyond the snow line of the host, as with the other four multiplanetary systems previously found by microlensing.
\end{abstract}

Key words: gravitational lensing: micro - planetary systems

\section{Introduction}

Detecting planetary systems with multiple planets located around and beyond the snow line is important for the investigation of the planet formation scenario. In planetary systems, snow line $a_{\text {snow }}$ indicates the distance from the central star at which the temperature is low enough for volatile compounds such as water, methane, carbon dioxide, and carbon monoxide to condense into solid ice grains. According to the standard theory of planet formation, i.e., core-accretion theory (Mizuno 1980; Stevenson 1982; Pollack et al. 1996), giant planets are thought to form around the snow line because the solid grains of water and other compounds rapidly accumulate into large planetary cores that will eventually grow into giant planets. The high efficiency of giant planet formation in this region may help the formation of multiple planets, as demonstrated by the existence of the two giant planets in the solar system, i.e., Jupiter and Saturn. Furthermore, there can be multiple snow lines corresponding to the individual compounds of protostar nebulae, and each snow line may be related to the formation of specific kinds of planets (Cleeves 2016). Around a Sun-like star, for example, the water snow line would roughly correspond to the orbit of Jupiter and the carbon monoxide snow line would approximately correspond to the orbit of Neptune. Therefore, estimating the occurrence rate of multiplanetary systems with cold planets will provide an important constraint on the planet formation mechanism.

Microlensing provides an important tool to detect multiplanetary systems with cold, wide-orbit planets. There currently exist 1325 planets in 657 known multiplanetary systems, at the time of writing this paper. ${ }^{42}$ Most transit planets and a majority of RV planets in multiplanetary systems are hot or warm planets located within the snow lines of the systems, and many of these planets are believed to have migrated from the place of their formation to their current locations via various dynamical mechanisms (Lin et al. 1996; Ward 1997; Murray et al. 1998).

\footnotetext{
38 MOA Collaboration.

39 OGLE Collaboration.

${ }^{40}$ KMTNet Collaboration.

${ }^{41}$ UKIRT Microlensing Team.

42 https://exoplanetarchive.ipac.caltech.edu/index.html
}

Microlensing, on the other hand, is sensitive to cold planets that are likely to have formed in situ and have not undergone largescale migration, and thus construction of an unbiased sample of planets in this region is important for the investigation of giant planet formation. The high sensitivity of the microlensing method to wide-orbit planets in multiple planetary systems is shown in the distribution of planets on the $a / a_{\text {snow }}-m_{\text {planet }}$ plane presented in Figure 1. Here, $a$ and $m_{\text {planet }}$ represent the semimajor axis and mass of the planet, respectively. Furthermore, the microlensing method does not rely on the luminosity of the host star. This makes the microlensing method a useful tool for investigating multiplanetary systems with faint host stars, for which the sensitivity of the other methods is low. This is particularly important for probing exoplanet populations, given that $\mathrm{M}$ dwarfs are the most common stars in the Galaxy.

There exist four reported multiple-planet systems detected using the microlensing method. The first system, OGLE-2006BLG-109L, is composed of a host with approximately half of the solar mass and two planets with masses of $\sim 0.71 M_{\mathrm{J}}$ and $\sim 0.27 M_{\mathrm{J}}$ and orbital separations of $\sim 2.3$ au and $\sim 4.6$ au, and thus the system resembles a scaled version of our solar system in that the mass ratio, separation ratio, and the equilibrium temperatures of the planets are similar to those of Jupiter and Saturn (Gaudi et al. 2008; Bennett et al. 2010). For OGLE2012-BLG-0026L, two planets with masses of $\sim 0.145 M_{\mathrm{J}}$ and $\sim 0.86 M_{\mathrm{J}}$ are orbiting a host star with a mass $\sim 1.06 M_{\odot}$ (Han et al. 2013; Beaulieu et al. 2016). For this system, there exist four degenerate solutions in the interpretation of the projected planet-host separations, but the separations of the individual planets are beyond the snow line in all solutions, being $\sim 4.0$ au and $\sim 4.8$ au for the best-fit solution. From the statistical arguments and dynamical analysis of the orbital configuration, Madsen \& Zhu (2019) argued that the two massive planets in OGLE-2012-BLG-0026L were likely in a resonance configuration. For OGLE-2014-BLG-1722L, two planets with masses of $\sim 0.18 M_{\mathrm{J}}$ and $\sim 0.27 M_{\mathrm{J}}$ are orbiting a late-type star with a mass of $\sim 0.4 M_{\odot}$. The projected separations from the host are $\sim 1.5$ au for the first planet and $\sim 1.7$ au or $\sim 2.7$ au for the second planet, and thus both planets are also located beyond the snow line (Suzuki et al. 2018). OGLE-2018-BLG-0532L is the most recently reported candidate system, in which two 


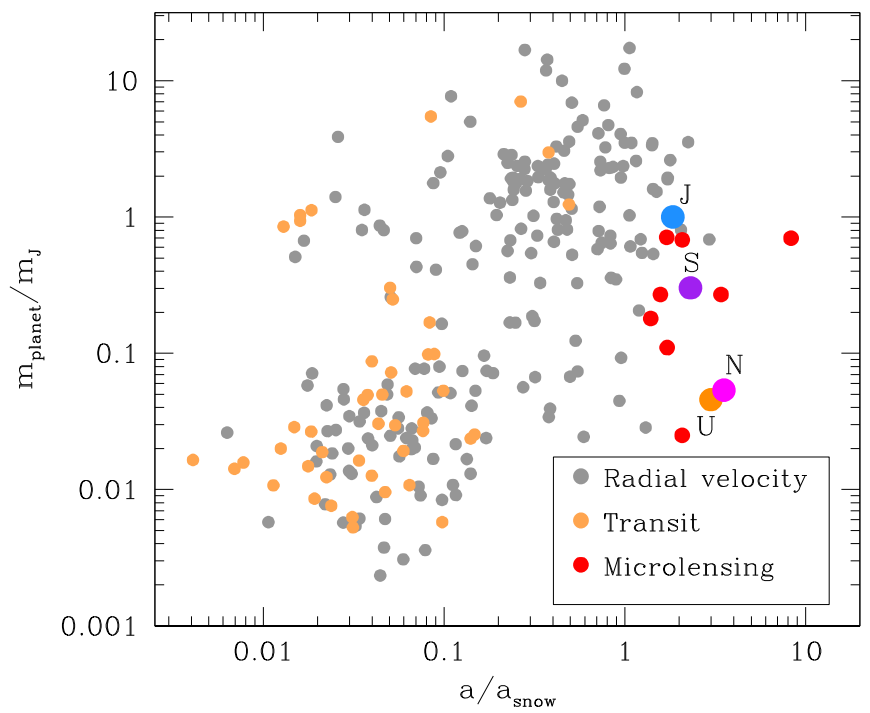

Figure 1. Distribution of planets in multiple planetary systems on the $a / a_{\text {snow }}$ $m_{\text {planet }}$ plane. Plot is based on 257 planets in 109 systems with known planet masses. Here, $a_{\text {snow }}$ represents the snow line. In order to compare these planets with those of the solar system, we present the locations of Jupiter ("J"), Saturn ("S"), Uranus ("U"), and Neptune ("N"). Colors of points indicate the detection methods that are marked in the inset.

planets with masses of $\sim 8 M_{\oplus}$ and $\sim 0.7 M_{\mathrm{J}}$ are located around an M-dwarf host having a mass of $\sim 0.25 M_{\odot}$ with projected separations from the host of $\sim 1.4$ au and 5.6 au, respectively (Ryu et al. 2019). From the detection efficiency analysis of the MOA (Suzuki et al. 2016) and $\mu$ FUN (Gould et al. 2010) surveys for the two microlensing multiplanetary systems OGLE-2006-BLG-109L and OGLE-2014-BLG-1722L, Suzuki et al. (2018) estimated that the occurrence rate of systems with multiple cold gas giant systems was $6 \% \pm 2 \%$. We note that the multiple-planet signatures in the lensing light curves are securely detected for OGLE-2006-BLG-109L and OGLE2012-BLG-0026L, but the signatures for OGLE-2014-BLG1722L and OGLE-2018-BLG-0532L are less reliable.

In this paper, we report a new multiple-planet system discovered by analyzing the combined data of the microlensing event OGLE-2018-BLG-1011 obtained by five lensing surveys. We describe data acquisition and processing in Section 2. In Sections 3 through 5, we describe the detailed procedure of analysis leading to the interpretation of the lens as a multipleplanet system. In Sections 6 and 7, we characterize the source of the event and estimate the physical parameters of the lens system, respectively. We summarize the results and conclude in Section 8.

\section{Observation and Data}

The source star of the microlensing event OGLE-2018-BLG1011 is located toward the Galactic bulge with equatorial coordinates (R.A., decl.) $)_{\mathrm{J} 2000}=(17: 56: 03.36,-29: 04$ : $58.4)$, which correspond to Galactic coordinates of $(l, b)=$ $(1.04,-2.04)$. In the middle panel of Figure 2, we present the $\sim 2.2$ yr baseline light curve observed by the OGLE survey. The top panel, showing the light curve for 100 days during $7800 \leqslant \mathrm{HJD}^{\prime} \equiv \mathrm{HJD}-2450000 \leqslant 7900$, is presented to check the short-term variability of the source brightness. It is found that the baseline magnitude, $I_{\text {base }} \sim 18.63$, is stable and the light curve does not show noticeable variability. We further
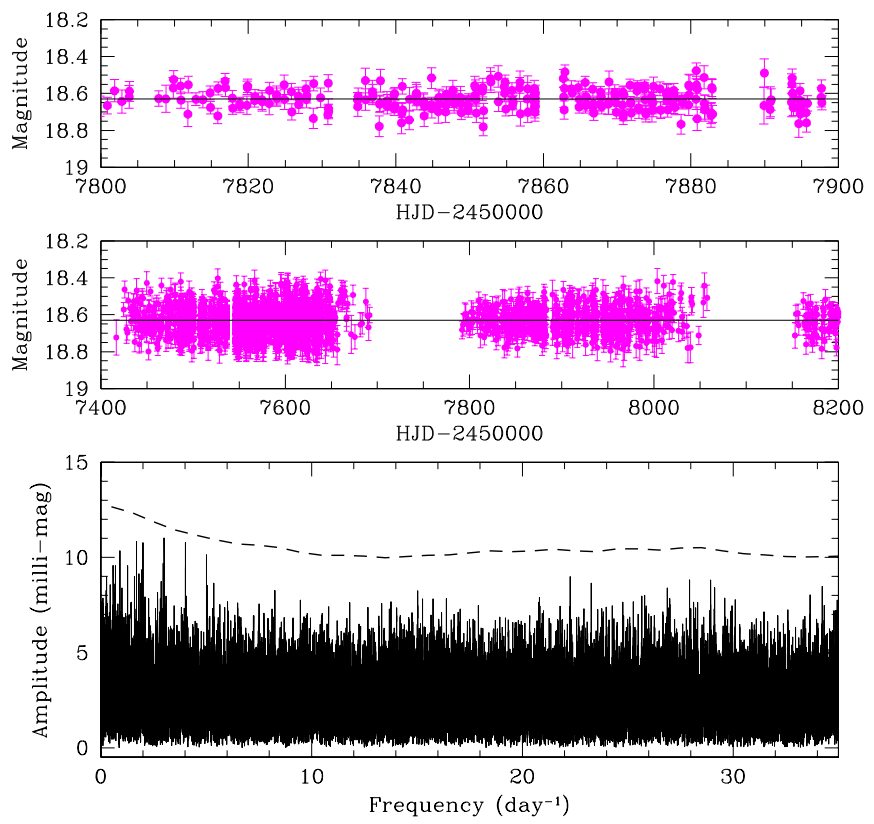

Figure 2. Baseline light curve. Upper and lower panels show the 100 day and $\sim 2.2 \mathrm{yr}$ baselines observed by the OGLE survey, respectively.

investigate the source variability by computing the power spectrum using the PERIOD04 code of Lenz \& Breger (2005). The power spectrum is presented in the bottom panel. The dashed line represents the limit with a signal-to-noise ratio $\mathrm{S} / N=4$, which is the empirically proposed threshold for variability (Breger et al. 1993). The spectrum shows no periodic variability greater than the imposed threshold, indicating that the source flux has been stable.

The brightening of the source star induced by lensing was found in the early rising stage of the event by the Optical Gravitational Lensing Experiment (OGLE; Udalski et al. $2015 \mathrm{~b}$ ), and the discovery was announced to the microlensing community on 2018 June $7\left(\mathrm{HJD}^{\prime} \sim 8277\right)$. The event was in the OGLE-IV BLG505.23 field that was monitored with a cadence of one day using the $1.3 \mathrm{~m}$ telescope located at the Las Campanas Observatory in Chile. Most OGLE data were acquired in the $I$ band, but some $V$-band images were obtained for the source color measurement.

The event was also located in the fields of two other major lensing surveys, namely the Microlensing Observations in Astrophysics (MOA; Bond et al. 2001; Sumi et al. 2003) and the Korea Microlensing Telescope Network (KMTNet; Kim et al. 2016). The MOA observations of the event were conducted in a customized broad $R$ band using the $1.8 \mathrm{~m}$ telescope located at the Mt. John Observatory in New Zealand. The cadence of the MOA survey was $\sim 10 /$ night in a survey mode, and reached up to $40 /$ night when the anomaly in the lensing light curve was in progress. The event was designated as MOA-2018-BLG-182 in the list of the "MOA Transient Alerts" page. ${ }^{43}$

The KMTNet survey utilizes three identical $1.6 \mathrm{~m}$ telescopes that are globally distributed for continuous coverage of lensing events. The individual telescopes are located at the Siding Spring Observatory in Australia (KMTA), Cerro Tololo Interamerican Observatory in Chile (KMTC), and the South African Astronomical Observatory in South Africa (KMTS).

43 http://www.massey.ac.nz/ iabond/moa/alert2018/alert.php 

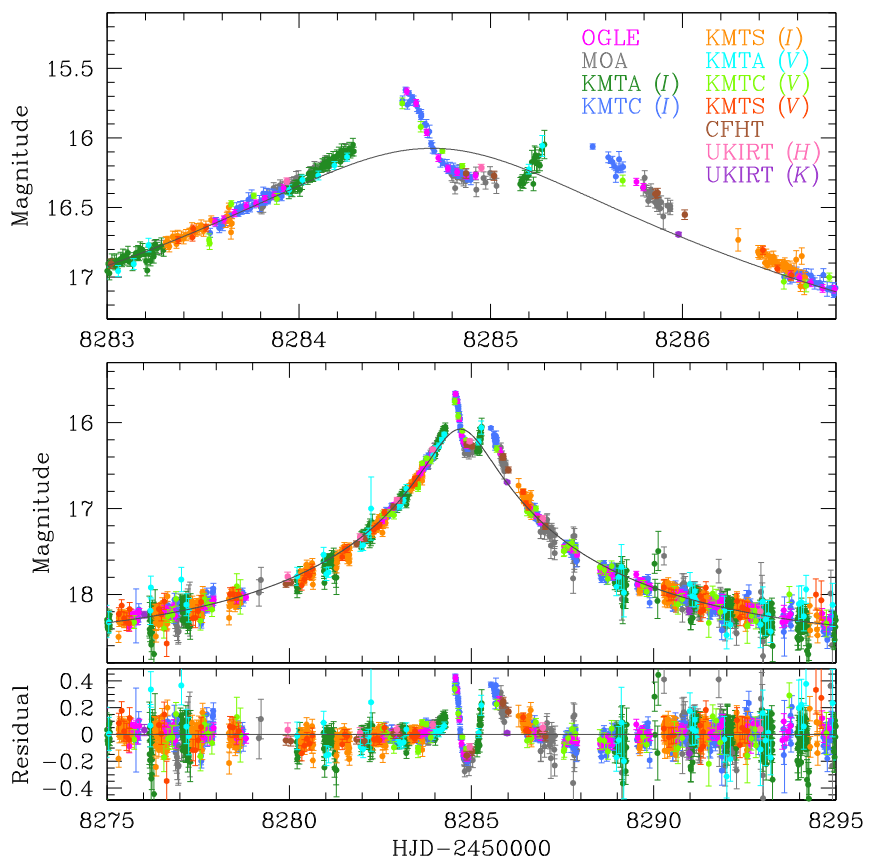

Figure 3. Light curve of the microlensing event OGLE-2018-BLG-1011. The upper panel shows the enlarged view of the anomaly region around the peak. The curve superposed on the data points is the model curve based on the pointsource point-lens interpretation.

The event was in the two overlapping fields (BLG02 and BLG042) of the KMTNet survey. Images were obtained mainly in the $I$ band, with occasional $V$-band data acquisition. We use $V$-band data not only for the source color measurement but also for the light curve analysis, to maximize the coverage of the short-term anomaly that appeared in the peak of the event light curve. The observational cadence of the KMTNet survey varied depending on the observation site, ranging from $4 / \mathrm{hr}$ for the KMTC telescope to $6 / \mathrm{hr}$ for the KMTA and KMTS telescopes. The event was detected by the KMTNet "event finder" (Kim et al. 2018) and was named KMT-2018-BLG-2122.

In addition to the three major microlensing surveys, the event was also observed by two lower-cadence surveys conducted by utilizing the Canada-France-Hawaii Telescope (CFHT; Zang et al. 2018) and the $3.8 \mathrm{~m}$ United Kingdom Infrared Telescope (UKIRT; Shvartzvald et al. 2017). The CFHT data include 62 points in the time range of $8256 \lesssim \mathrm{HJD}^{\prime} \lesssim 8346$. The UKIRT observations were conducted with a daily cadence in the $H$ band and occasional observations in the $K$ band. The CFHT data include 62 points obtained during $8256 \lesssim \mathrm{HJD}^{\prime} \lesssim 8346$, and the UKIRT data include $49 \mathrm{H}$-band and $10 \mathrm{~K}$-band data points acquired during $7864 \lesssim \mathrm{HJD}^{\prime} \lesssim 8336$ and $7882 \lesssim \mathrm{HJD}^{\prime} \lesssim$ 8329 , respectively.

Data sets obtained by the OGLE and MOA surveys were released almost in real time through the "Early Warning System" 44 and "MOA Transient Alerts" pages. This in turn facilitated monitoring of the real-time evolution of the event. On 2018 June $16\left(\mathrm{HJD}^{\prime} \sim 8285.5\right)$, D. Suzuki of the MOA group noticed a deviation of the light curve from a point-source point-lens model and issued an anomaly alert. With this alert, the MOA group increased their observation cadence. See the light curve of the event and the anomaly around the peak presented in Figure 3. The alert triggered real-time analysis of

\footnotetext{
${ }^{44}$ http://ogle.astrouw.edu.pl/ogle4/ews/ews.html
}

the light curve and a series of models - all of which were based on binary-lens interpretations-were released by V. Bozza, A. Cassan, D. Bennett, and Y. Hirao during the progress of the anomaly. Although results were not circulated, the first author of this paper (C. Han) was also conducting analysis of the event with the progress of the event. When the anomaly ended, however, it was found that none of these models could fully explain the observed anomaly. The fact that the real-time analyses done by five people using separate, independently written software packages reached the same conclusion (that a binary-lens interpretation cannot properly explain the observed data) strongly suggests the necessity of an interpretation of the event that is different from the binary-lens explanation.

We note that there are gaps in the data during the anomaly despite the coverage by five surveys. The gaps centered at $\mathrm{HJD}^{\prime} \sim 8284.4$ and 8285.4 could have been covered by the KMTS telescope located in Africa, and the gap centered at $\mathrm{HJD}^{\prime} \sim 8286.2$ could have been observed by the KMTA telescope located in Australia. Unfortunately, no data could be obtained because of poor weather conditions at the respective sites.

In our analysis of the event, we use photometry data processed with the codes developed by the individual survey groups: OGLE by Woźniak (2000), MOA by Bond et al. (2001), and KMTNet by Albrow (2009). All of these codes are based on the difference imaging technique of Alard \& Lupton (1998). For a subset of KMTNet data, additional photometry is processed using the pyDIA photometry (Albrow 2009) to measure the source color. For the use of multiple data sets obtained by different groups and processed by using different photometry codes, we normalize the photometric measurement uncertainties of the data sets following the procedure of Yee et al. (2012), in which the photometric uncertainties are rescaled by

$$
\sigma=k\left(\sigma_{0}^{2}+\sigma_{\min }^{2}\right)^{1 / 2} .
$$

The quadratic term $\sigma_{0}$ is added so that the cumulative distribution of $\chi^{2}$ ordered by magnification is approximately linear. This process ensures the dispersion of data points will be consistent with the error bars. The coefficient " $k$ " is a factor used for rescaling the errors so that $\chi^{2}$ per degree of freedom $\left(\chi^{2} /\right.$ dof $)$ for each data set becomes unity. The latter process is needed to prevent each data set from being under- or overrepresented, compared to other data sets. In Table 1, we present the values of $k$ and $\sigma_{0}$.

\section{Binary-lens (2L1S) Interpretations}

The light curve of the event exhibits an anomaly that is characterized by two bumps near the peak. We start the analysis of the anomaly with the " $2 \mathrm{~L} 1 \mathrm{~S}$ " interpretation, in which the event is produced by a lens composed of two masses, "2L," deflecting the light from a single source, "1S."

It is known that a double-bump feature in the peak region of a lensing light curve can be produced by two major channels of 2L1S events (Han \& Gaudi 2008). One channel is the case in which the source trajectory approaches the two neighboring cusps of the central caustic produced by a binary lens composed of similar masses with a projected separation between the lens components significantly smaller (close binary) or wider (wide binary) than the Einstein radius $\theta_{\mathrm{E}}$ of the lens system. The other channel is the case in which the source approaches the back-end cusp of the central caustic produced by a planetary lens system 
Table 1

Photometric Uncertainty Rescaling Parameters

\begin{tabular}{lcc}
\hline \hline Data Set & $k$ & $\sigma_{0}(\mathrm{mag})$ \\
\hline OGLE & 1.170 & 0.020 \\
MOA & 1.490 & 0.020 \\
KMTA $(I$, BLG02) & 1.523 & 0.020 \\
KMTA $(I$, BLG42) & 2.170 & 0.020 \\
KMTC $(I$, BLG02) & 1.086 & 0.030 \\
KMTC $(I$, BLG42) & 1.584 & 0.010 \\
KMTS $(I$, BLG02) & 1.396 & 0.020 \\
KMTS $(I$, BLG42) & 1.261 & 0.030 \\
KMTA $(V$, BLG02) & 2.017 & 0.010 \\
KMTA $(V$, BLG42) & 2.135 & 0.010 \\
KMTC $(V$, BLG02) & 1.470 & 0.025 \\
KMTC $(V$, BLG42) & 1.421 & 0.005 \\
KMTS $(V$, BLG02) & 1.288 & 0.010 \\
KMTS $(V$, BLG42) & 1.253 & 0.010 \\
CFHT & 0.806 & 0.040 \\
UKIRT $(H)$ & 0.500 & 0.030 \\
UKIRT $(K)$ & 0.224 & 0.030 \\
\hline
\end{tabular}

Note. The notations in the parentheses of the KMTNet data sets represent the passbands and fields of observation.

with a mass ratio between the lens components $q \ll 1$ and a starplanet separation similar to $\theta_{\mathrm{E}}$ (Choi et al. 2012; Park et al. 2014; Bozza et al. 2016). We refer to the former and latter channels as the "binary" and "planetary" channels, respectively.

For the 2L1S analysis, we first conduct a dense grid search for the lensing parameters $s$ and $q$, which represent the binary separation normalized to $\theta_{\mathrm{E}}$ and the mass ratio between the lens components, respectively. Considering that central perturbations can be produced either by a planetary companion with a low mass ratio or a binary companion with very wide or close separations from the primary, we set the ranges of $s$ and $q$ for the grid search wide enough to check both the planetary and binary solutions. The initial grid search is done in the ranges of $-1.0 \leqslant \log s \leqslant 1.0$ and $-4.0 \leqslant \log q \leqslant 1.0$ for the separation and mass ratio, respectively, with 50 grids for each range. We then identify local minima in the distribution of $\Delta \chi^{2}$ on the $s-q$ plane. For the individual local solutions, we gradually narrow down the ranges of the parameter space in the grid search. Apart from these lensing parameters, a basic 2L1S modeling requires additional parameters, including the time of the closest lens-source approach, $t_{0}$, the lens-source separation at that time, $u_{0}$ (normalized to $\theta_{\mathrm{E}}$ ), the Einstein timescale, $t_{\mathrm{E}}$, and the source trajectory angle with respect to the binary-lens axis, $\alpha$. In the case when the source crosses the caustic formed by the binary lens, the lensing light curve is affected by finite-source effects and one needs an additional parameter of $\rho$, which represents the source radius $\theta_{*}$ normalized to $\theta_{\mathrm{E}}$, i.e., $\rho=\theta_{*} / \theta_{\mathrm{E}}$, to account for these effects. In computing finite-source magnifications, we consider surface-brightness variation of the source stars caused by the limb darkening. The stellar type of the source turns out to be a G-type turn-off star (Section 6), and thus we adopt a linear limb-darkening coefficient of $\Gamma_{I}=0.5$. For a given set of $(s, q)$, we search for the other lensing parameters using a downhill approach based on the Markov Chain Monte Carlo (MCMC) method.

In Figure 4, we present the distribution of $\Delta \chi^{2}$ from the bestfit model on the $s-q$ parameter plane obtained from the initial grid search. We find that there exist four local solutions. For one pair of the local solutions, the mass ratios are $q \sim 1$, and

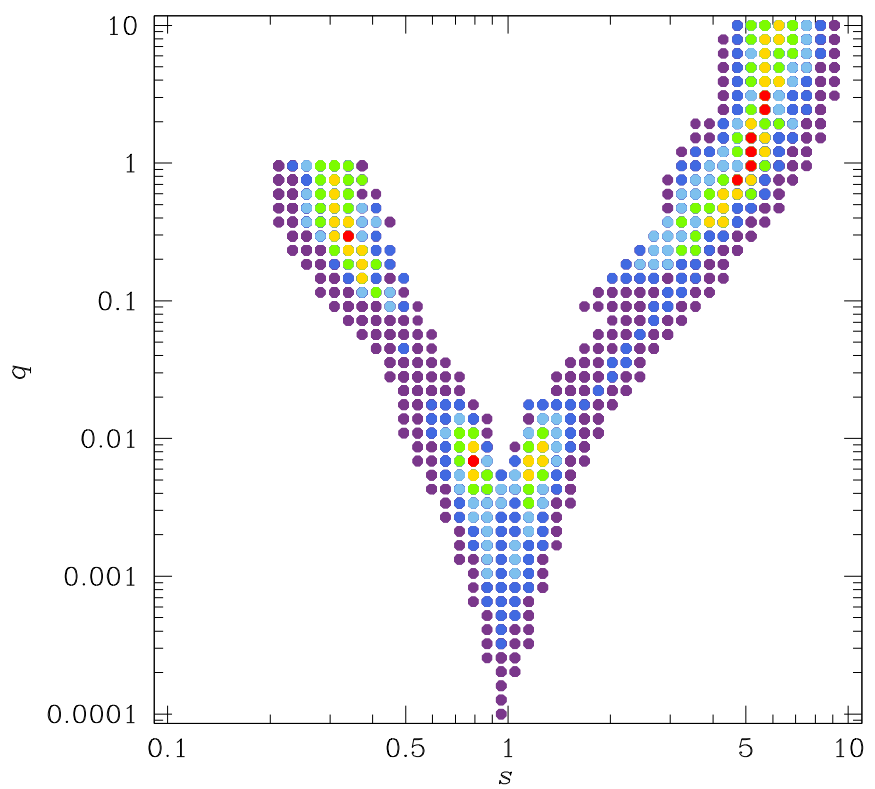

Figure 4. The $\Delta \chi^{2}$ distribution on the plane of the binary-lensing parameters $s$ and $q$. Color coding indicates points within $1 n \sigma$ (red), $2 n \sigma$ (yellow), $3 n \sigma$ (green), $4 n \sigma$ (cyan), $5 n \sigma$ (blue), and $6 n \sigma$ (purple) with respect to the best-fit value and $n=10$

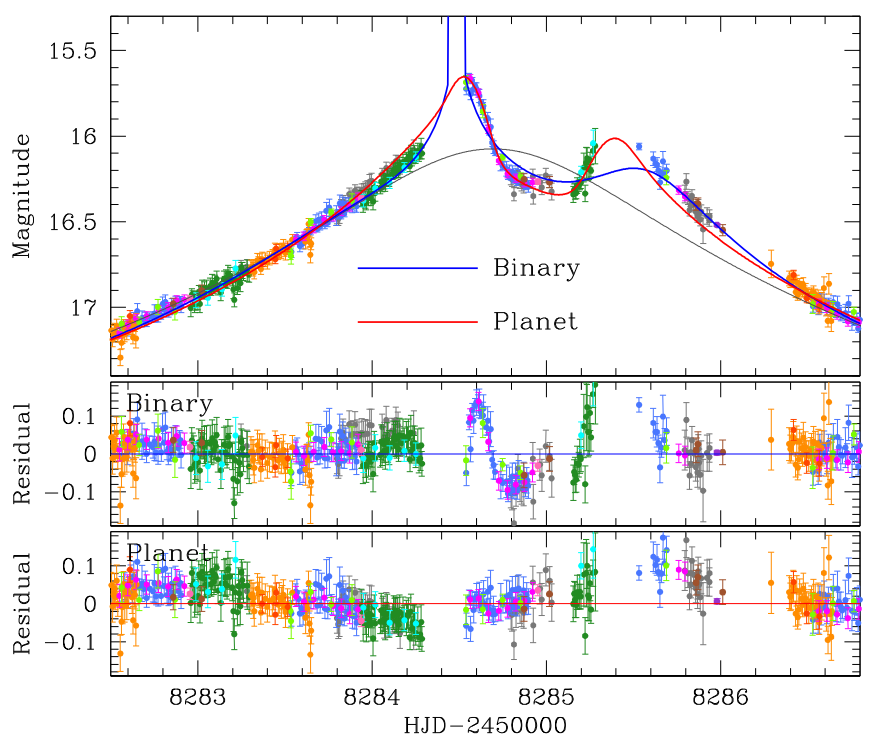

Figure 5. Model light curves of the two solutions obtained for the 2L1S interpretation: binary (blue curve: $s<1.0$ (close solution); see Table 3) and planetary (red curve: $s<1.0$ (close solution); see Table 3) solutions. The two lower panels show the residuals from the individual models. The lens-system configurations corresponding to the individual solutions are presented in Figure 6.

thus the solutions correspond to binary-lens solutions. For the other pair, the mass ratios are $q<10^{-2}$, indicating that they correspond to planetary-mass lens solutions. For each pair, we find that there exist two solutions with $s<1$ (close solution) and $s>1$ (wide solution), which are generated by the close/ wide degeneracy (Griest \& Safazadeh 1998; Dominik 1999).

In Figure 5, we present the model light curves corresponding to the "binary" (blue curve) and "planetary" (red curve) 2L1S solutions superposed on the observed data, together with the residuals from the models (presented in the lower two panels). 
Table 2

Comparison of Models

\begin{tabular}{|c|c|c|c|}
\hline & Solution & & $\chi^{2}$ \\
\hline \multirow[t]{4}{*}{$2 \mathrm{~L} 1 \mathrm{~S}$} & binary & $s<1.0$ & 8545.6 \\
\hline & & $s>1.0$ & 8470.6 \\
\hline & planetary & $s<1.0$ & 8439.7 \\
\hline & & $s>1.0$ & 8477.0 \\
\hline \multicolumn{3}{|l|}{$1 \mathrm{~L} 2 \mathrm{~S}$} & 10853.6 \\
\hline \multicolumn{3}{|l|}{$2 \mathrm{~L} 2 \mathrm{~S}$} & 8047.3 \\
\hline \multirow[t]{10}{*}{$3 \mathrm{~L} 1 \mathrm{~S}$} & Planet-binary & $s_{2}<1.0, s_{3}<1.0$ & 7825.4 \\
\hline & & $s_{2}<1.0, s_{3}>1.0$ & 7825.5 \\
\hline & & $s_{2}>1.0, s_{3}<1.0$ & 7865.2 \\
\hline & & $s_{2}>1.0, s_{3}>1.0$ & 7882.9 \\
\hline & Multiple-planet (I) & $s_{2}<1.0$ & 7783.8 \\
\hline & & $s_{2}>1.0$ & 7790.7 \\
\hline & Multiple-planet (II) & $s_{2}<1.0, s_{3}<1.0$ & 7718.0 \\
\hline & & $s_{2}<1.0, s_{3}>1.0$ & 7761.6 \\
\hline & & $s_{2}>1.0, s_{3}<1.0$ & 7717.7 \\
\hline & & $s_{2}>1.0, s_{3}>1.0$ & 7756.5 \\
\hline
\end{tabular}

Note. For the $3 \mathrm{~L} 1 \mathrm{~S}$ solutions, $s_{2}$ and $s_{3}$ represent the respective normalized separations between $M_{1}-M_{2}$ and $M_{1}-M_{3}$, where $M_{1}$ represents the primary lens and $M_{2}$ and $M_{3}$ denote the companions.

We note that the individual solutions are further refined based on the local minima obtained from the grid search by allowing all lensing parameters, including $s$ and $q$, to vary. In Table 2, we compare the $\chi^{2}$ values of the four 2 L1S solutions. In Table 3 , we list the best-fit lensing parameters of the individual 2L1S solutions. We also note that the model light curves corresponding to the close and wide solutions of each of the binary and planetary solution pairs are similar to each other, and thus Figure 5 presents the one yielding a better fit to the data. In Figure 6, we present the lens-system configurations, which represent the source trajectory with respect to the caustic, of the binary (upper panel) and planetary (lower panel) solutions. We note that the presented configurations are for the close $(s<1.0)$ solutions. From the fits of the individual models to the observed data, it is found that the binary-lens solution leaves substantial residuals in the region $8284.5 \lesssim \mathrm{HJD}^{\prime} \lesssim 8285.6$, and the planetary solution leaves residuals in the region $8285.6 \lesssim$ $\mathrm{HJD}^{\prime} \lesssim 8286.0$. These residuals indicate that neither the binary nor the planetary 2L1S solutions adequately describe the observed data, and therefore suggest that a new interpretation of the light curve is required.

We additionally check whether the fit further improves by considering the microlens-parallax (Gould 1992) and/or the lens-orbital (Dominik 1998; Ioka et al. 1999) effects. The improvement yielded by these higher-order effects is negligible, mainly due to the short timescale, $t_{\mathrm{E}} \sim 12$ days, of the event.

\section{Binary-source (1L2S and 2L2S) Interpretation}

Gaudi (1998) pointed out the possibility that a short-term perturbation, which was the main feature of a planetary microlensing signal produced by major-image perturbations, could be reproduced by a subset of binary-source events, in which a single-mass lens, $1 \mathrm{~L}$, passed close to the fainter member of the binary source, $2 \mathrm{~S}$. The degeneracy between 2L1S and 1L2S interpretations can often be severe, as demonstrated in the cases of OGLE-2002-BLG-055 (Gaudi \& Han 2004), MOA-2012-BLG-486 (Hwang et al. 2013), and OGLE-2014-BLG-1186 (Dominik et al. 2019). In addition to the short-term anomaly considered by Gaudi (1998), it was shown that the $2 \mathrm{~L} 1 \mathrm{~S} / 1 \mathrm{~L} 2 \mathrm{~S}$ degeneracy could extend to various cases of planetary lens system configurations from the analysis of anomalies in the cases of OGLE-2016-BLG-0733 by Jung et al. (2017) and KMT-2017-BLG-0962 and KMT-2017-BLG1119 by Shin et al. (2019).

We check the binary-source interpretation of the anomaly in the light curve of OGLE-2018-BLG-1011 by conducting a 1L2S modeling of the observed light curve. The addition of the source companion requires the inclusion of additional parameters in modeling. These parameters are $t_{0,2}, u_{0,2}$, and $q_{F}$, which represent the time of the closest lens approach to the source companion, the impact parameter to the companion, and the flux ratio between the source stars, respectively. We note that finite-source effects are considered in the modeling. From this modeling, we find that the 1L2S solution provides a poorer fit than the best-fit 2L1S solutions, by $\Delta \chi^{2}=2313.1$ and 2418.9 compared to the binary and planetary $2 \mathrm{~L} 1 \mathrm{~S}$ solutions, respectively, and thus we reject the solution. In Figure 7, we present the best-fit 1L2S model (dotted curve superposed on the data points).

We also check the model in which both the source and lens are binaries, 2L2S model. Figure 7 shows the model light curve (solid curve) of the best-fit 2L2S solution. In Figure 8, we also present the lens-system configuration corresponding to the model. We note that there are two source trajectories, because the source is a binary in the $2 \mathrm{~L} 2 \mathrm{~S}$ model. The solution provides a better fit than the 2L1S and 1L2S solutions, but it leaves noticeable residuals in the region 8282.5 $\lesssim \mathrm{HJD}^{\prime} \lesssim 8283.2$. As we will show in the following section, the fit of the 2L2S solution is worse than the best-fit solution based on other interpretation by $\Delta \chi^{2}=329.6$. In Table 2 , we list the $\chi^{2}$ values of the 1L2S and 2L2S solutions. In Table 3, we also list the best-fit lensing parameters of the 1L2S and 2L2S solutions.

\section{Triple-lens (3L1S) Interpretation}

Knowing the inadequacy of the 2L1S, 1L2S, and 2L2S solutions in describing the observed light curve, we then model the light curve assuming a triple-lens interpretation in which the lens contains three components, "3L." We try 3L1S modeling because the solutions obtained from the 2L1S modeling partially describe the observed central perturbation, and the residual from the $2 \mathrm{~L} 1 \mathrm{~S}$ model may be explained by introducing an additional lens component.

Central perturbations of lensing light curves in 3L1S cases can be produced through two major channels. One channel is through multiple-planet systems, in which the individual planets located in the lensing zone can affect the magnification pattern of the central perturbation region (Gaudi et al. 1998). Among the four known multiplanetary systems detected by microlensing, three systems (OGLE-2006-BLG-109Lb,c, OGLE-2012-BLG-0026Lb,c, and OGLE-2018-BLG-0532Lab) were detected through the central perturbations induced by two planets. The other channel is through planet+binary systems. Similar to the central caustic produced by a planet, a very close or a very wide binary companion can also induce a small caustic in the central magnification region and thus can affect the magnification pattern. Among the four known 
Table 3

Lensing Parameters of 2L1S, 1L2S, and 2L2S Models

\begin{tabular}{|c|c|c|c|c|c|c|}
\hline \multirow{2}{*}{ Parameter } & \multicolumn{2}{|c|}{ 2L1S (Binary) } & \multicolumn{2}{|c|}{ 2L1S (Planet) } & \multirow{2}{*}{$1 \mathrm{~L} 2 \mathrm{~S}$} & \multirow{2}{*}{$2 \mathrm{~L} 2 \mathrm{~S}$} \\
\hline & $(s<1)$ & $(s>1)$ & $(s<1)$ & $(s>1)$ & & \\
\hline$\overline{\chi^{2}}$ & 8546.6 & 8470.6 & 8439.7 & 8477.0 & 10853.6 & 8047.3 \\
\hline$u_{0,1}$ & $0.032 \pm 0.001$ & $0.022 \pm 0.001$ & $0.062 \pm 0.001$ & $0.063 \pm 0.001$ & $0.061 \pm 0.001$ & $0.063 \pm 0.001$ \\
\hline$t_{0,2}\left(\mathrm{HJD}^{\prime}\right)$ & $\cdots$ & $\cdots$ & $\cdots$ & $\cdots$ & $8285.686 \pm 0.011$ & $8285.403 \pm 0.007$ \\
\hline$u_{0,2}$ & $\cdots$ & $\cdots$ & $\cdots$ & $\cdots$ & $0.027 \pm 0.001$ & $0.011 \pm 0.001$ \\
\hline$q$ & $0.301 \pm 0.015$ & $1.108 \pm 0.096$ & $(6.98 \pm 0.15) \times 10^{-3}$ & $(7.03 \pm 0.17) \times 10^{-3}$ & $\cdots$ & $(6.61 \pm 0.09) \times 10^{-3}$ \\
\hline$\alpha(\mathrm{rad})$ & $0.246 \pm 0.005$ & $0.235 \pm 0.005$ & $4.434 \pm 0.005$ & $4.440 \pm 0.005$ & $\cdots$ & $4.253 \pm 0.008$ \\
\hline$\rho_{1}\left(10^{-3}\right)$ & $\cdots$ & $\cdots$ & $\cdots$ & $\cdots$ & $\cdots$ & $\cdots$ \\
\hline$\rho_{2}\left(10^{-3}\right)$ & $\cdots$ & $\cdots$ & $\cdots$ & $\cdots$ & $7.58 \pm 3.07$ & $0.80 \pm 0.13$ \\
\hline$q_{F, I}$ & $\cdots$ & $\cdots$ & $\cdots$ & $\cdots$ & $0.24 \pm 0.01$ & $0.071 \pm 0.003$ \\
\hline
\end{tabular}

Note. HJD' = HJD-2450000.
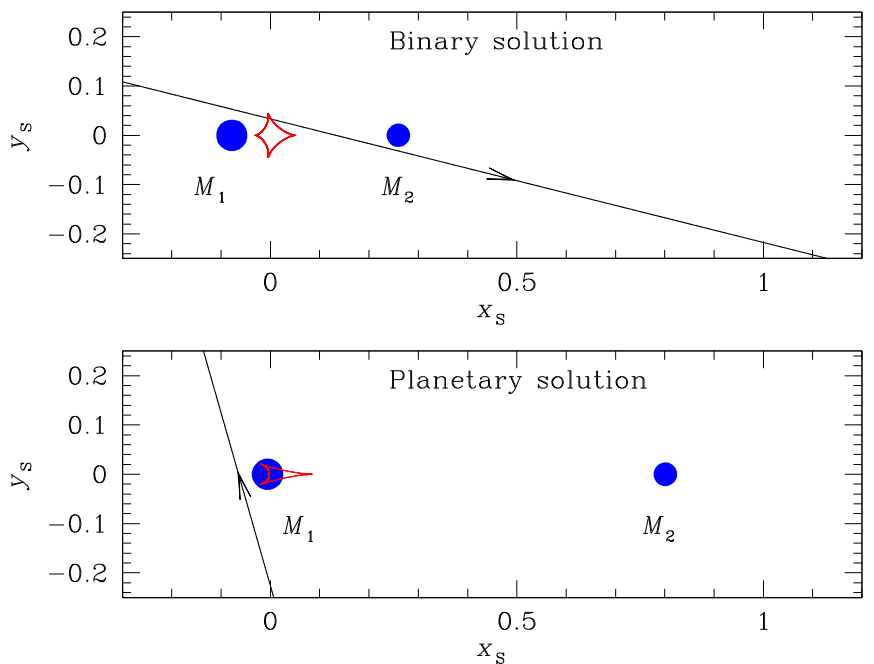

Figure 6. Lens-system configurations of the $2 \mathrm{~L} 1 \mathrm{~S}$ solutions. The upper and lower panels correspond to the "binary" (with $s<1.0$ ) and "planetary" (with $s<1.0)$ solutions, respectively. In each panel, the line with an arrow represents the source trajectory and the red close curve is the caustic. The blue dots marked by $M_{1}$ and $M_{2}$ denote the positions of the binary lens components.

microlensing planetary systems in binaries, ${ }^{45}$ the circumbinary planetary system OGLE-2007-BLG-349L(AB)c was detected through this channel.

The lensing behavior of triple-lens systems is qualitatively different from that of binary-lens systems, resulting in complex caustic structures, such as nested caustic and self-intersections. The range of the critical-curve topology and the caustic structure of the triple lens has not yet been fully explored, making it difficult to analyze triple-lens events (Daněk \& Heyrovský 2015, 2019). As a result, there are some events suspected to be triple-lens events, but for which plausible models have yet not been proposed: e.g., OGLE-2008-BLG-270, OGLE-2012-BLG-0442/MOA2012-BLG-245, OGLE-2012-BLG-0207/MOA-2012-BLG-105, and OGLE-2018-BLG-0043/MOA-2018-BLG-033. In some cases, interpretations of triple-lens events can be confused with

45 OGLE-2007-BLG-349L(AB)c (Bennett et al. 2016), OGLE-2016-BLG0613LABb (Han et al. 2017), OGLE-2008-BLG-092LABb (Poleski et al. 2014), and OGLE-2013-BLG-0341LAbB (Gould et al. 2014).

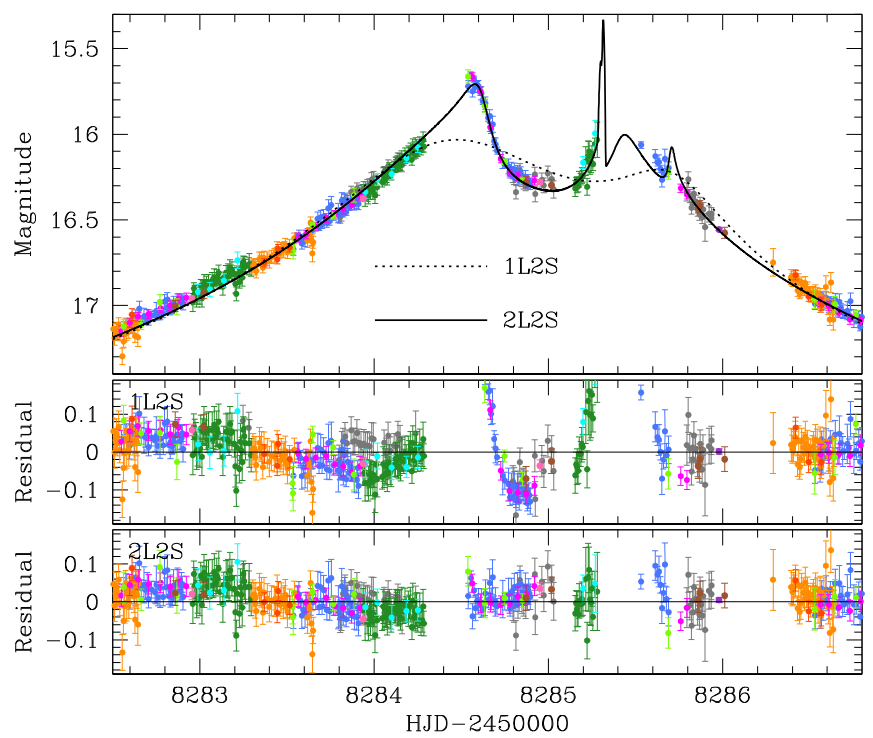

Figure 7. Model light curves obtained for the 1L2S (dotted curve) and 2L2S (solid curve) interpretations. The two lower panels show the residuals of the individual models.

those of binary-lens events, as in the cases of MACHO-97-BLG41 (Bennett et al. 1999; Albrow et al. 2000; Jung et al. 2013) and OGLE-2013-BLG-0723 (Udalski et al. 2015a; Han et al. 2016).

Despite the diversity and complexity of the lensing behavior, triple-lens events can be readily analyzed for some specific lens cases. One such case occurs when the lens companions produce small perturbations with minor interference in the magnification pattern between the perturbations produced by the individual companions. In this case, the resulting anomaly can be approximated by the superposition of the two binary anomalies, in which the individual primary-companion pairs act as independent two-body systems (Han 2005). This binarysuperposition approximation can be applied for two cases of triple-lens systems, in which the lens is composed of multiple planets, as demonstrated in the cases of OGLE-2006-BLG-109 (Gaudi et al. 2008), OGLE-2012-BLG-0026 (Han et al. 2013), and planets in close or wide binary systems, as demonstrated in the case of OGLE-2016-BLG-0613 (Han et al. 2017). The 2L1S modeling of OGLE-2018-BLG-1011 yields two local 


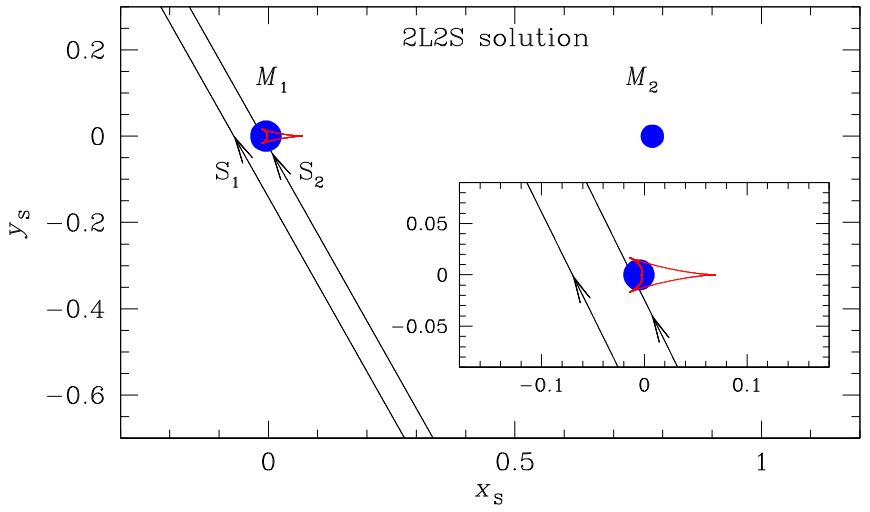

Figure 8. Lens-system configuration of the best-fit 2L2S model. Notations are same as those in Figure 6, except that there are two source trajectories because the source is a binary. The trajectories marked by $S_{1}$ and $S_{2}$ represent those of the brighter and fainter source stars, respectively.

solutions, with a planetary companion and a close/wide binary companion, respectively. This suggests that the event may be the case for which the analysis based on the superposition approximation is valid.

The 3L1S modeling is done in two steps. In the first step, we conduct a grid search for the separation $s_{3}$ and mass ratio $q_{3}$ between $M_{1}$ and $M_{3}$, as well as the orientation angle $\psi$ of the third body with respect to the $M_{1}-M_{2}$ axis. Here, we use the subscripts "1"-" 3 " to denote the individual lens components. In this search, we fix the values of $\left(s_{2}, q_{2}, \alpha\right)$ as those obtained from the $2 \mathrm{~L} 1 \mathrm{~S}$ modeling. Because two local 2L1S solutions are found, i.e., the "binary" and "planetary" solutions, we conduct two sets of modeling with the initial values of $\left(s_{2}, q_{2}, \alpha\right)$ of the "binary" and "planetary" solutions obtained from the 2L1S modeling. We use the parameters of the close 2L1S solution as the initial parameters, but the result would not be affected with the use of the wide solution parameters, because of the similarity between the model light curves of the close and wide solutions. In Figure 9, we present the $\Delta \chi^{2}$ map in the $s_{3}-q_{3}$ parameter plane obtained from the grid search with the initial values of the "planetary" $2 \mathrm{~L} 1 \mathrm{~S}$ solution. In the second step, we refine the solutions found from the grid search by allowing all parameters to vary. See Bennett (2010) in which a similar approach of triple-lensing modeling is described.

The 3L1S modeling yields three sets of solutions. One set of solutions results from the starting values of $\left(s_{2}, q_{2}, \alpha\right)$ of the binary 2L1S solution and the other two sets result from the starting $\left(s_{2}, q_{2}, \alpha\right)$ values of the planetary $2 \mathrm{~L} 1 \mathrm{~S}$ solution. The solutions found based on the binary $2 \mathrm{~L} 1 \mathrm{~S}$ solution indicates that the lens is a planetary system in a binary. We designate these solutions as the "planet-binary" solutions. The two sets of solutions found based on the planetary 2L1S solution indicate that the lens is a multiplanetary system. We designate these two sets of solutions as the "multiple-planet (I)" and "multipleplanet (II)" solutions. For all 3L1S solutions, the gross features of the anomalies are better described and the fits greatly improve with respect to the $2 \mathrm{~L} 1 \mathrm{~S}$ and $1 \mathrm{~L} 2 \mathrm{~S}$ models. In Table 2, we list the $\chi^{2}$ values of the individual solutions. We discuss the details of the individual solutions in the following subsections.

\subsection{Planet-binary Solution}

We find the "planet-binary" 3L1S solutions from the modeling using the initial values of $\left(s_{2}, q_{2}, \alpha\right)$ obtained from the "binary" 2L1S solution. We find four degenerate solutions

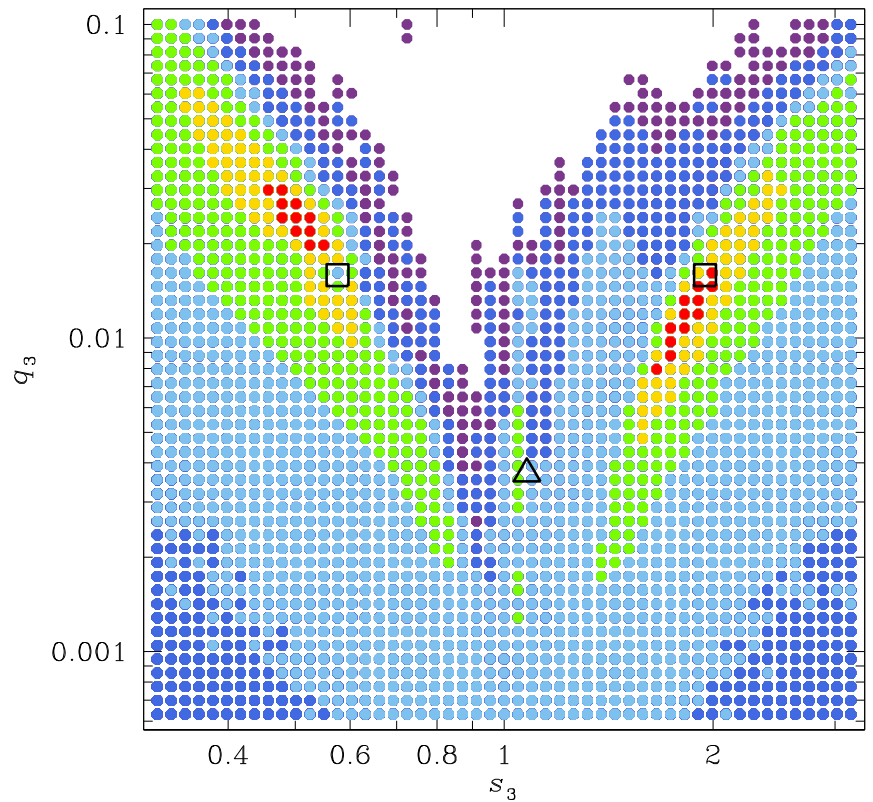

Figure 9. The $\Delta \chi^{2}$ map in the $s_{3}-q_{3}$ parameter plane obtained from a grid search with the initial values of the "planetary" 2L1S solution. The location marked by the empty triangle corresponds to the "multiple-planet (I)" solution, and the two marked by the empty squares corresponds to the "multiple-planet (II)" solutions. The color coding is same as that of Figure 4.

resulting from the close/wide degeneracy in the separations between $M_{1}-M_{2}\left(s_{2}<1.0\right.$ and $\left.s_{2}>1.0\right)$ and $M_{1}-M_{3}\left(s_{3}<1.0\right.$ and $\left.s_{3}>1.0\right)$ pairs. In Table 4, we list the lensing parameters of the individual solutions along with $\chi^{2}$ values. The solutions with $s_{2}<1.0$ are preferred over the solutions with $s_{2}>1.0$ by $\Delta \chi^{2} \geq 40$. For the two solutions with $s_{2}<1.0$, however, the degeneracy between the solutions with $s_{3}<1.0$ and $s_{3}>1.0$ is very severe, i.e., $\Delta \chi^{2} \sim 0.5$.

In Figure 10, we present the model light curve (blue dashed curve) of the best-fit planet-binary solution (with $s_{2}<1.0$ and $\left.s_{3}<1.0\right)$ and the residual from the model. In Figure 11, we also present the lens-system configuration corresponding to the solution. We note that the central caustic structures of the other degenerate solutions are similar to the one presented. According to the planet-binary solutions, the source trajectory passed the upper tip of the central caustic, producing two caustic-crossing spikes at HJD $\sim 8284.3$ (caustic entrance) and 8284.5 (caustic exit), but the crossings happened in the region of the data ending just before the caustic entrance and starting just after the caustic exit. See the enlarged view of the crossing-crossing region of the light curve presented in the lower middle inset of the top panel in Figure 10. As a result, the value of the normalized source radius $\rho$ cannot be securely measured and its value is not presented in Table 4.

A comparison of the lens-system configuration of the "planetbinary" 3L1S solution (presented in Figure 11) to that of the "binary" 2L1S solution (presented in the upper panel in Figure 6) reveals that a tiny, wedge-shaped caustic appears, due to the additional planetary companion with a mass ratio of $q_{3} \sim(1.7-2.8) \times 10^{-3}$. A further comparison with the "binary" 2L1S model (presented in Figure 5) shows that the introduction of the third body substantially reduces the residuals of the $2 \mathrm{~L} 1 \mathrm{~S}$ solution in the region $8284.5 \lesssim \mathrm{HJD}^{\prime} \lesssim 8285.1$ and improves the fit by $\Delta \chi^{2} \sim 645$. We find that the fit improvements offered by the microlens-parallax and lens-orbital effects are negligible. 
Table 4

Lensing Parameters of "Planet-binary" 3L1S Solutions

\begin{tabular}{lcccc}
\hline \hline Parameter & $s_{2}<1.0, s_{3}<1.0$ & $s_{2}<1.0, s_{3}>1.0$ & $s_{2}>1.0, s_{3}<1.0$ & $s_{2}>1.0, s_{3}>1.0$ \\
\hline$\chi^{2}$ & 7825.4 & 7825.5 & 7865.2 & 7882.9 \\
$t_{0}\left(\mathrm{HJD}^{\prime}\right)$ & $8284.557 \pm 0.006$ & $8284.564 \pm 0.005$ & $8284.557 \pm 0.004$ & $8284.549 \pm 0.003$ \\
$u_{0}$ & $0.033 \pm 0.001$ & $0.034 \pm 0.001$ & $0.023 \pm 0.001$ & $0.023 \pm 0.001$ \\
$t_{\mathrm{E}}($ days $)$ & $12.80 \pm 0.14$ & $12.70 \pm 0.15$ & $17.12 \pm 0.20$ & $4.872 \pm 0.073$ \\
$s_{2}$ & $0.357 \pm 0.004$ & $0.354 \pm 0.004$ & $0.977 \pm 0.079$ & $4.559 \pm 0.12$ \\
$q_{2}$ & $0.263 \pm 0.012$ & $0.273 \pm 0.012$ & $0.268 \pm 0.004$ & $0.703 \pm 0.035$ \\
$\alpha(\mathrm{rad})$ & $0.282 \pm 0.0054$ & $0.283 \pm 0.005$ & $0.896 \pm 0.025$ & $0.265 \pm 0.004$ \\
$s_{3}$ & $0.812 \pm 0.015$ & $1.262 \pm 0.023$ & $(1.71 \pm 0.26) \times 10^{-3}$ & $(2.28 \pm 0.26) \times 10^{-3}$ \\
$q_{3}$ & $(2.81 \pm 0.45) \times 10^{-3}$ & $1.299 \pm 0.002$ & $1.371 \pm 0.005$ & $1.361 \pm 0.004$ \\
$\psi(\mathrm{rad})$ & $1.259 \pm 0.006$ & & & \\
\hline
\end{tabular}

Note. HJD' = HJD-2450000.

\subsection{Multiple-planet (I) Solution}

The "multiple-planet (I)" 3L1S solution set is one of the two sets of solutions obtained using the starting values of $\left(s_{2}, q_{2}, \alpha\right)$ from the "planetary" 2L1S solution. For this set of solutions, we find two degenerate solutions caused by the close/wide degeneracy in estimating the $M_{1}-M_{2}$ separation, $s_{2}$. The projected separation between $M_{1}$ and $M_{3}$ is very close to unity, $s_{3} \sim 1.08$, and thus there is no close/wide degeneracy in estimating $s_{3}$. The solution with $s_{2}<1.0$ is slightly preferred over the solution with $s_{2}>1.0$ by $\Delta \chi^{2}=6.9$. Both companions $M_{2}$ and $M_{3}$ have planetary mass ratios of $q_{2} \sim 6.5 \times 10^{-3}$ and $q_{3} \sim 3.7 \times 10^{-3}$, respectively, indicating that the lens is a multiplanetary system composed of two giant planets. In Table 5, we list the lensing parameters for both solutions with $s_{2}<1.0$ and $s_{2}>1.0$. The "multiple-planet (I)" solution with $s<1.0$ provides a better fit than the planetary planetary $2 \mathrm{~L} 1 \mathrm{~S}$ solution by $\Delta \chi^{2}=555.9$.

In Figure 10, we plot the model light curve of the "multipleplanet (I)" solution with $s_{2}<1.0$ (red dotted curve) and the residuals from the model. In Figure 12, we also present the lens-system configurations corresponding to the solution. The third body $M_{3}$ induces a resonant caustic in the central magnification region in addition to the central caustic induced by $M_{2}$; the solution with $s_{2}>1.0$ results in a similar central caustic. In the inset of Figure 12, we mark the positions of the source corresponding to the two major bumps at $t_{1}=\mathrm{HJD}^{\prime} \sim$ 8284.6 and $t_{2} \sim 8285.4$. The first bump at $t_{1}$ is produced when the source approaches the back-end cusp of the caustic induced by $M_{2}$, while the second bump at $t_{2}$ is produced by the source approach close to one of the back-end cusps of the caustic induced by $M_{3}$. The source also approaches another cusp induced by $M_{3}$ at the position marked by $t^{\prime} \sim 8283.0$ in the inset of Figure 12. This approach also produces a bump, although the bump is weak; see the upper left inset in the top panel of Figure 10. We note that, unlike the binary-planet solution, the "multiple-planet (I)" solution explains the anomaly without a caustic-crossing feature. As a result, and similar to the binary-planet solution, the normalized source radius $\rho$ cannot be measured and it is not presented in Table 5. Similar to the case of the binary-planet solution, the higher-order effects are not important for the description of the observed light curve.

\subsection{Multiple-planet (II) Solution}

For given $\left(s_{2}, q_{2}, \alpha\right)$ values of the "planetary" $2 \mathrm{~L} 1 \mathrm{~S}$ solution, we find another set of $3 \mathrm{~L} 1 \mathrm{~S}$ solutions. We designate these solutions as the "multiple-planet (II)" solutions. The mass ratios between $M_{1}$ and $M_{3}$ of the "multiple-planet (II)" solutions, $q_{3} \sim 15 \times 10^{-3}$, are considerably greater than the mass ratios of the "multiple-planet (I)" solutions, $\sim 3.7 \times 10^{-3}$. In addition, the $M_{1}-M_{3}$ separations of the "multiple-planet (II)" solutions are substantially different from unity, while the $s_{3}$ values of the "multiple-planet (I)" solutions are close to unity. The best-fit "multiple-planet (II)" solution (with $s_{2}>1.0$ and $s_{3}<1.0$ ) yields a better fit to the observed data than the planetary $2 \mathrm{~L} 1 \mathrm{~S}$ solution by $\Delta \chi^{2}=722.0$.

In Table 6, we list the lensing parameters of the "multipleplanet (II)" solutions. We find that there exist four solutions resulting from the close/wide degeneracies in both $s_{2}$ and $s_{3}$. Comparison of the $\chi^{2}$ values of the individual solutions shows that the solutions with $s_{3}<1.0$ are favored over the solutions with $s_{3}>1.0$ by $\Delta \chi^{2} \sim 40$. However, the two solutions resulting from the close/wide degeneracy in $s_{2}$ are very severe, with $\Delta \chi^{2}=0.3$. In Figure 10, we present the model light curve of the solution with $s_{2}>1.0$ and $s_{3}<1.0$, which yields the best fit to the data, and the residual from the model.

In Figure 13, we present the lens-system configuration of the best-fit "multiple-planet (II)" solution. The central caustic appears to be the superposition of the two sets of central caustics induced by the $M_{1}-M_{2}$ and $M_{1}-M_{3}$ two-body lens pairs. According to this solution, the bumps at $t_{1}$ and $t_{2}$ are produced by the successive approaches of the source close to the back-end cusps of the central caustic produced by the $M_{1}-M_{2}$ binary pair. However, the cusp of the first source approach is deformed by $M_{3}$, and thus the central caustic is different from that of the $M_{1}-M_{2}$ binary. We note that the patterns of central caustics for the other degenerate solutions are similar to the one presented.

For the "multiple-planet (II)" solutions, the source does not cross the caustic, but finite-source effects are securely detected - unlike the previous two sets of $3 \mathrm{~L} 1 \mathrm{~S}$ solutions. To investigate the reason for this, we construct the magnification pattern around the central caustic. Figure 14 shows the constructed magnification contour map, in which the innermost contour is drawn at $A=50$ and the other contours are drawn at the descending magnifications with a step $\Delta A=2$ from the center outward. The line with an arrow represents the source trajectory, and the two orange circles on the trajectory represent the source locations at the times of the first $\left(t_{1}\right)$ and second $\left(t_{2}\right)$ caustic approaches, respectively. The size of the circle is scaled to the source size. We find that the magnification on the surface of the source varies substantially and this results in deviation of 


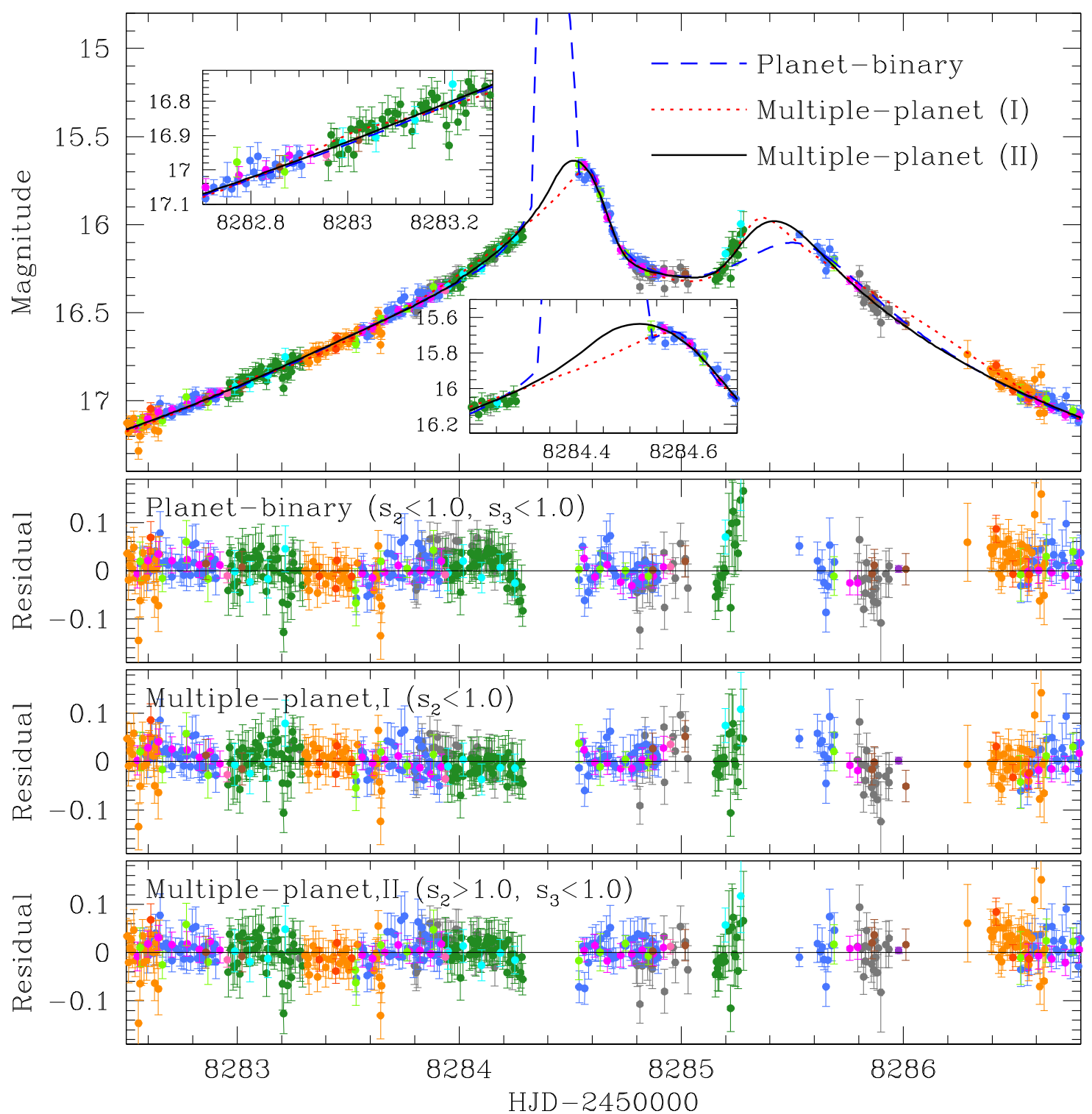

Figure 10. Model light curves of the three solutions obtained for the 3L1S interpretation: "planet-binary" (blue curve: $s_{2}<1, s_{3}<1$; see, Table 4), "multipleplanet (I)" (red curve: $s_{2}<1$; see, Table 5), "multiple-planet (II)" (black curve: $s_{2}>1, s_{3}<1$; see, Table 6). The three lower panels show the residuals from the individual models. The upper left inset in the top panel shows the enlarged view of the source star's cusp approach at around $\mathrm{HJD}^{\prime} \sim 8283.0$ according to the "multiple-planet (I)" solution, and the lower middle inset shows the zoom of the caustic-crossing region according to the "planet-binary" solution.

the light curve from the point-source light curve during the times around both bumps. The normalized source radius is securely measured from the deviation of the light curve; although there is some variation depending on the solution, the measured normalized source radius is $\rho \sim 0.012$. In Figure 15, we present the distribution of points in the MCMC chain on the $u_{0}-\rho$ plane for the best-fit "multiple-planet (II)" solution. Higher-order effects are not detected for the solution.

\subsection{Comparison of Models}

Because the overall features of the observed central perturbation are described by three sets of 3L1S solutions, we closely investigate the individual solutions. For this, we construct a cumulative distribution of $\Delta \chi^{2}=\chi_{\mathrm{p}-\mathrm{b}}^{2}-\chi_{\mathrm{m}-\mathrm{p}}^{2}$ for the data around the region of the anomaly. Here, the subscripts "p-b" and "m-p" denote "planet-binary" and "multiple-planet" solutions, respectively. In Figure 16, we present the constructed cumulative $\Delta \chi^{2}$ distributions.
From the cumulative $\Delta \chi^{2}$ distributions, together with the residuals of the three solutions presented in Figure 10, we find that the "multiple-planet (II)" solution provides a better fit than the other two solutions do. Compared to the "planet-binary" solution, the "multiple-planet (II)" solution better explains the rising part of the light curve in the region of the anomaly at $\mathrm{HJD}^{\prime} \sim 8285.2$, resulting in a better fit by $\Delta \chi^{2}=107.7$ over the "planet-binary" solution. The "planet-binary" solution is additionally disfavored by Occam's razor because the major features occur only during the gap in the data. Compared to the "multiple-planet (I)" solution, the "multiple-planet (II)" solution better describes the light curve in the region 8282.0 $\lesssim \mathrm{HJD}^{\prime} \lesssim 8284.1$, resulting in a better fit by $\Delta \chi^{2}=65.9$. We note that, according to the "multiple-planet (I)" solution, the source approached a cusp of the caustic induced by $M_{3}$ at around $t^{\prime} \sim 8283.0$, producing a weak bump. However, no such a bump is expected according to the "multiple-planet (II)" solution, and that makes this solution fit the data better than the "multiple-planet (I)" solution does. 


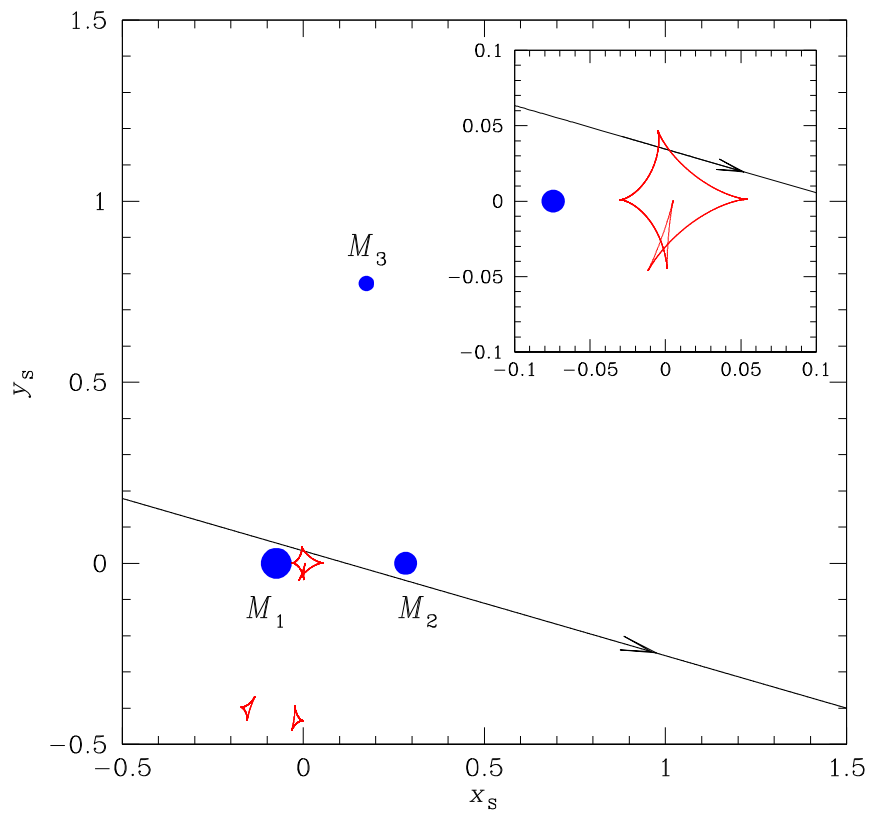

Figure 11. Lens-system configuration of the "planet-binary" 3L1S solution with $s_{2}<1.0$ and $s_{3}<1.0$. The blue dots marked by $M_{1}, M_{2}$, and $M_{3}$ represent the locations of the three lens components. The inset shows the enlargement of the central caustic region. We note that the central caustic structures of the other three degenerate solutions presented in Table 4 are similar to the one presented.

Table 5

Lensing Parameters of "Multiple-planet (I)" 3L1S Solutions

\begin{tabular}{lcc}
\hline \hline Parameter & $s_{2}<1.0$ & $s_{2}>1.0$ \\
\hline$\chi^{2}$ & 7783.8 & 7790.7 \\
$t_{0}\left(\mathrm{HJD}^{\prime}\right)$ & $8284.761 \pm 0.004$ & $8284.756 \pm 0.004$ \\
$u_{0}$ & $0.070 \pm 0.001$ & $0.072 \pm 0.001$ \\
$t_{\mathrm{E}}($ days $)$ & $10.30 \pm 0.11$ & $10.37 \pm 0.11$ \\
$s_{2}$ & $0.786 \pm 0.003$ & $1.193 \pm 0.005$ \\
$q_{2}\left(10^{-3}\right)$ & $6.47 \pm 0.14$ & $6.60 \pm 0.16$ \\
$\alpha(\mathrm{rad})$ & $4.424 \pm 0.006$ & $4.424 \pm 0.006$ \\
$s_{3}$ & $1.078 \pm 0.005$ & $1.076 \pm 0.004$ \\
$q_{3}\left(10^{-3}\right)$ & $3.70 \pm 0.15$ & $3.75 \pm 0.14$ \\
$\psi(\mathrm{rad})$ & $5.175 \pm 0.006$ & $5.174 \pm 0.006$ \\
\hline
\end{tabular}

Note. HJD' $=$ HJD-2450000.

Considering the better description of the detailed structures of the lensing light curve over the other models, we conclude that the "multiple-planet (II)" solution provides the most plausible model of the observed data.

\section{Source Star}

Characterizing a source star in microlensing is important in order to estimate the angular Einstein radius, $\theta_{\mathrm{E}}$, which is related to the angular source radius $\theta_{*}$ by

$$
\theta_{\mathrm{E}}=\frac{\theta_{*}}{\rho} .
$$

For OGLE-2018-BLG-1011, the normalized source radius is securely measured even though the source does not cross the caustic. Next, one needs to estimate the angular source radius in order to estimate the angular Einstein radius.

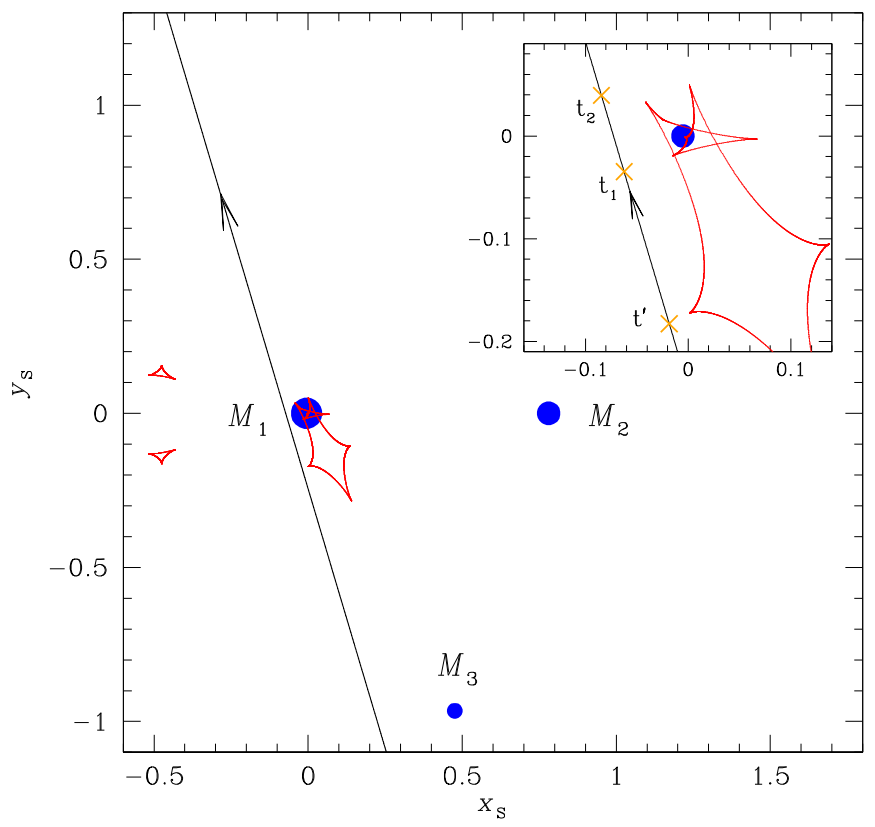

Figure 12. Lens-system configuration of the "multiple-planet (I)" 3L1S solution with $s_{2}<1.0$. Notations are same as those in Figure 11. The three "X" marks in the inset represent the source positions at the times of the cusp approaches at $t_{1}=8284.6, t_{2}=8285.4$, and $t^{\prime}=8283.0$.

For the estimation of the angular source radius, we first estimate the dereddened color $(V-I)_{\mathrm{S}, 0}$ and brightness $I_{\mathrm{S}, 0}$ of the source star. For this estimation, we use the Yoo et al. (2004) method, in which the color and magnitude are calibrated using the centroid of the red giant clump (RGC) in the colormagnitude diagram (CMD) as a reference. In Figure 17, we present the location of the source in the CMD of stars within $\sim 20^{\prime}$ around the source star. The CMD is constructed based on the pyDIA photometry of the KMTC $I$ - and $V$-band data sets. The instrumental color and brightness of the source are $(V-I$, $I)_{\mathrm{S}}=(1.42 \pm 0.01,19.93 \pm 0.01)$. The offsets in color and magnitude of the source with respect to the RGC centroid, which is located at $(V-I, I)_{\mathrm{RGC}}=(1.71,16.99)$, are $\Delta(V-I$, $I)_{\mathrm{S}}=(-0.29,2.95)$. With the known dereddened values of the RGC centroid of $(V-I, I)_{\mathrm{RGC}, 0}=(1.06,14.38)$ (Bensby et al. 2011; Nataf et al. 2013), the dereddened color and brightness of the source star are then estimated as $(V-I, I)_{\mathrm{S}, 0}=(V-I, I)_{\mathrm{S}}+$ $\Delta(V-I, I)_{\mathrm{S}}=(0.77 \pm 0.01,17.33 \pm 0.01)$. These values indicate that the source is a late G-type turn-off star. In Figure 17, we also mark the position of the blend in the CMD. It is found that the blend has a color and a brightness that are similar to those of the source. The brightness of the blend is $I_{b}=19.04 \pm 0.01$ as measured in the OGLE photometry system, which is approximately calibrated.

With the known dereddened color and brightness, the angular source radius is estimated first by converting the $V-I$ color into the $V-K$ color using the color-color relation (Bessell \& Brett 1988) and second using the $(V-K) / \theta_{*}$ relation (Kervella et al. 2004). This procedure yields an angular source radius of

$$
\theta_{*}=1.98 \pm 0.08 \mu \text { as. }
$$

We note that two major factors affect the precision of the estimated angular source radius. The first is the uncertainty of the dereddened color, $\sim 0.05 \mathrm{mag}$, and the other is the uncertainty in the position of RGC, $\sim 0.1 \mathrm{mag}$. The uncertainty 
Table 6

Lensing Parameters of "Multiple-planet (II)" 3L1S Solutions

\begin{tabular}{lcccc}
\hline \hline Parameter & $s_{2}<1.0, s_{3}<1.0$ & $s_{2}<1.0, s_{3}>1.0$ & $s_{2}>1.0, s_{3}<1.0$ & $s_{2}>1.0, s_{3}>1.0$ \\
\hline$\chi^{2}$ & 7718.0 & 7761.6 & 7717.7 & 7756.5 \\
$t_{0}\left(\mathrm{HJD}^{\prime}\right)$ & $8284.818 \pm 0.005$ & $8284.868 \pm 0.009$ & $8284.801 \pm 0.005$ & $8284.851 \pm 0.009$ \\
$u_{0}$ & $0.049 \pm 0.001$ & $0.047 \pm 0.001$ & $0.053 \pm 0.001$ & $0.052 \pm 0.001$ \\
$t_{\mathrm{E}}($ days $)$ & $12.19 \pm 0.14$ & $12.41 \pm 0.21$ & $12.42 \pm 0.15$ & $1.281 \pm 0.009$ \\
$s_{2}$ & $0.750 \pm 0.005$ & $0.747 \pm 0.007$ & $9.84 \pm 0.26$ & $1.276 \pm 0.011$ \\
$q_{2}\left(10^{-3}\right)$ & $9.25 \pm 0.21$ & $9.73 \pm 0.30$ & $4.360 \pm 0.008$ & $10.22 \pm 0.37$ \\
$\alpha(\mathrm{rad})$ & $4.361 \pm 0.008$ & $4.433 \pm 0.008$ & $0.582 \pm 0.005$ & $4.430 \pm 0.008$ \\
$s_{3}$ & $0.577 \pm 0.005$ & $1.954 \pm 0.048$ & $15.00 \pm 0.61$ & $1.929 \pm 0.047$ \\
$q_{3}\left(10^{-3}\right)$ & $15.24 \pm 0.59$ & $16.06 \pm 1.70$ & $4.858 \pm 0.009$ & $4.740 \pm 0.011$ \\
$\psi(\mathrm{rad})$ & $4.859 \pm 0.010$ & $4.733 \pm 0.011$ & $12.13 \pm 0.71$ & $11.72 \pm 0.75$ \\
$\rho\left(10^{-3}\right)$ & $11.26 \pm 0.72$ & $11.29 \pm 0.76$ & & \\
\hline
\end{tabular}

Note. HJD' $=$ HJD-2450000.

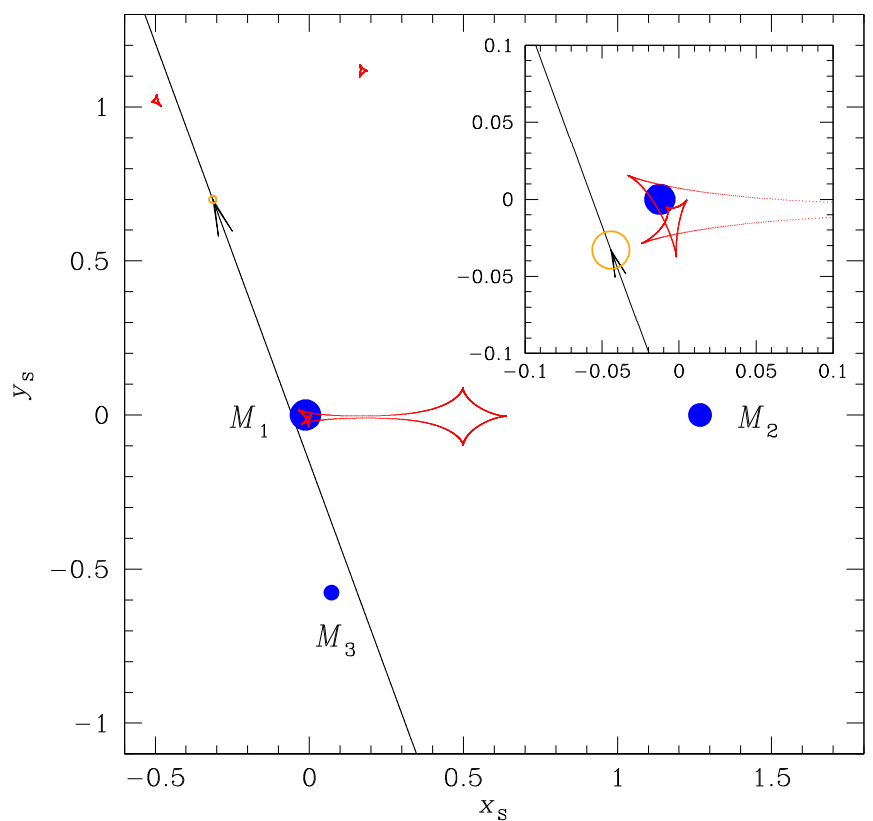

Figure 13. Lens-system configuration of the "multiple-planet (II)" 3L1S solution with $s_{2}>1.0$ and $s_{3}<1.0$. Notations are same as those in Figure 11. The small empty circle in the inset represents the source; it is presented to show the scaled size of the source relative to the caustic.

of $\theta_{*}$ is estimated by considering the combined uncertainty, which is $\sim 7 \%$, of these two factors (Gould 2014b). With the measured $\theta_{*}$ together with $\rho$, the angular Einstein radius is estimated as

$$
\theta_{\mathrm{E}}=\frac{\theta_{*}}{\rho}=0.09 \pm 0.01 \text { mas. }
$$

With the angular Einstein radius combined with the event timescale, the relative lens-source proper motion is estimated as

$$
\mu=\frac{\theta_{\mathrm{E}}}{t_{\mathrm{E}}}=2.81 \pm 0.22 \text { mas. }
$$

In Table 7, we summarize the colors and magnitudes of the source and blend, as well as the estimated angular source radius, Einstein radius, and relative lens-source proper motion.

For an independent constraint on the source star distance, we check the source information in the list of Gaia data release 2

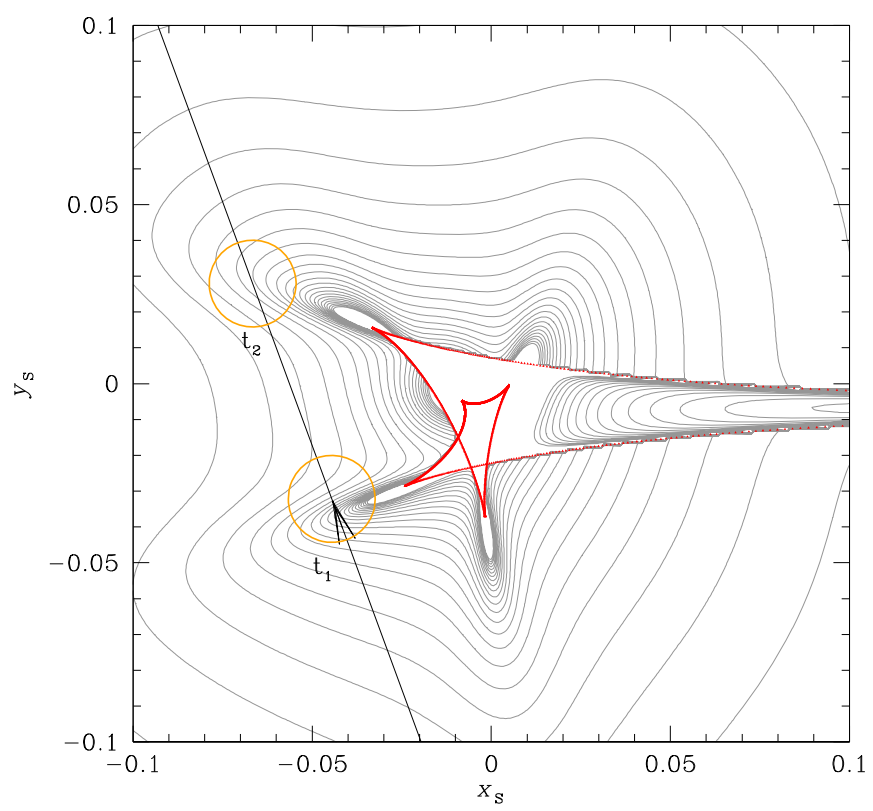

Figure 14. Magnification pattern around the central caustic of the "multipleplanet (II)" solution with $s_{2}>1.0$ and $s_{3}<1.0$. The innermost contour is drawn at $A=50$, and the other contours are drawn at the descending magnifications with a step $\Delta A=2$ from the center outward. The two empty circles represent the source locations at the times of the two major bumps at $t_{1}=8284.6$ and $t_{2}=8285.4$, and the size of the circle is scaled to the source size.

(Gaia DR2; Gaia Collaboration et al. 2018). However, there is no information regarding the absolute parallax and proper motion of the source, because its $G$-band magnitude of $G=19.76$ is fainter than the Gaia limit of $G \sim 18$. As a result, it is difficult to constrain the source distance from the Gaia data.

\section{Physical Parameters}

For the unique determination of the mass, $M$, and distance to the lens, $D_{\mathrm{L}}$, one needs to measure both the angular Einstein radius $\theta_{\mathrm{E}}$ and the microlens parallax $\pi_{\mathrm{E}}$, which are related to $M$ and $D_{\mathrm{L}}$ by the relations

$$
M=\frac{\theta_{\mathrm{E}}}{\kappa \pi_{\mathrm{E}}} ; \quad D_{\mathrm{L}}=\frac{\mathrm{au}}{\pi_{\mathrm{E}} \theta_{\mathrm{E}}+\pi_{\mathrm{S}}},
$$




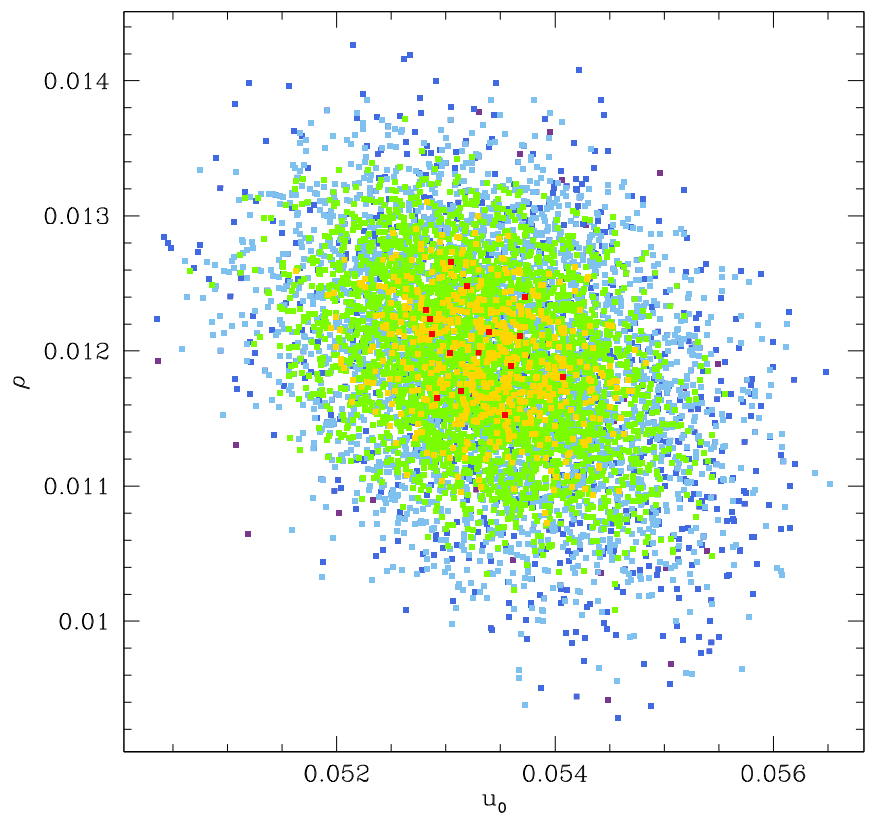

Figure 15. Distribution of points in the MCMC chain on the $u_{0}-\rho$ plane. The red, yellow, green, cyan, and blue colors represent points with $1 \sigma, 2 \sigma, 3 \sigma, 4 \sigma$, and $5 \sigma$, respectively.

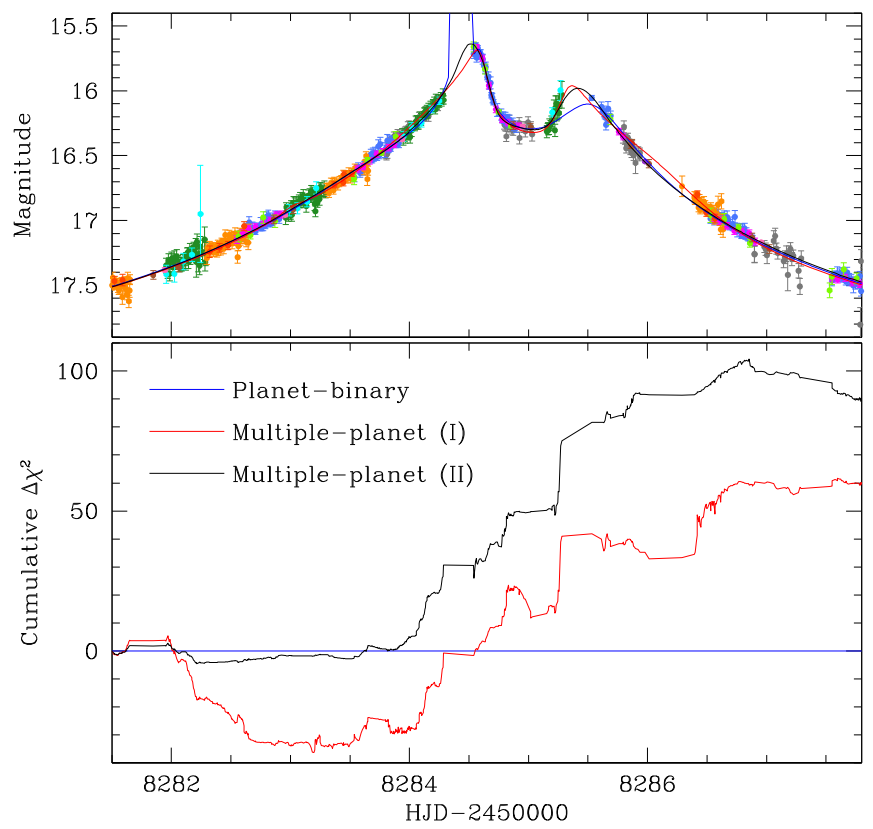

Figure 16. Cumulative distribution of $\Delta \chi^{2}=\chi_{\mathrm{p}-\mathrm{b}}^{2}-\chi_{\mathrm{m}-\mathrm{p}}^{2}$ between the planet-binary and "multiple-planet" 3L1S solutions for the data in the regions of the anomaly. Here, the subscripts "p-b" and "m-p" denote planet-binary and "multiple-planet" solutions, respectively. The light curve in the upper panel is presented to show the regions of $\chi^{2}$ difference.

where $\kappa=4 G /\left(c^{2} \mathrm{au}\right), \pi_{\mathrm{S}}=\mathrm{au} / D_{\mathrm{S}}$, and $D_{\mathrm{S}}$ is the source distance-which is $\sim 8 \mathrm{kpc}$ for a source star located in the bulge. For OGLE-2018-BLG-1011, the angular Einstein radius is measured, but the microlens parallax is not. Therefore, we estimate the physical lens parameters by conducting Bayesian analysis with the constraints of the measured $t_{\mathrm{E}}$ and $\theta_{\mathrm{E}}$.

A microlensing Bayesian analysis requires prior models of the lens mass function and the physical and dynamical distributions of the Galaxy. We adopt the mass function of

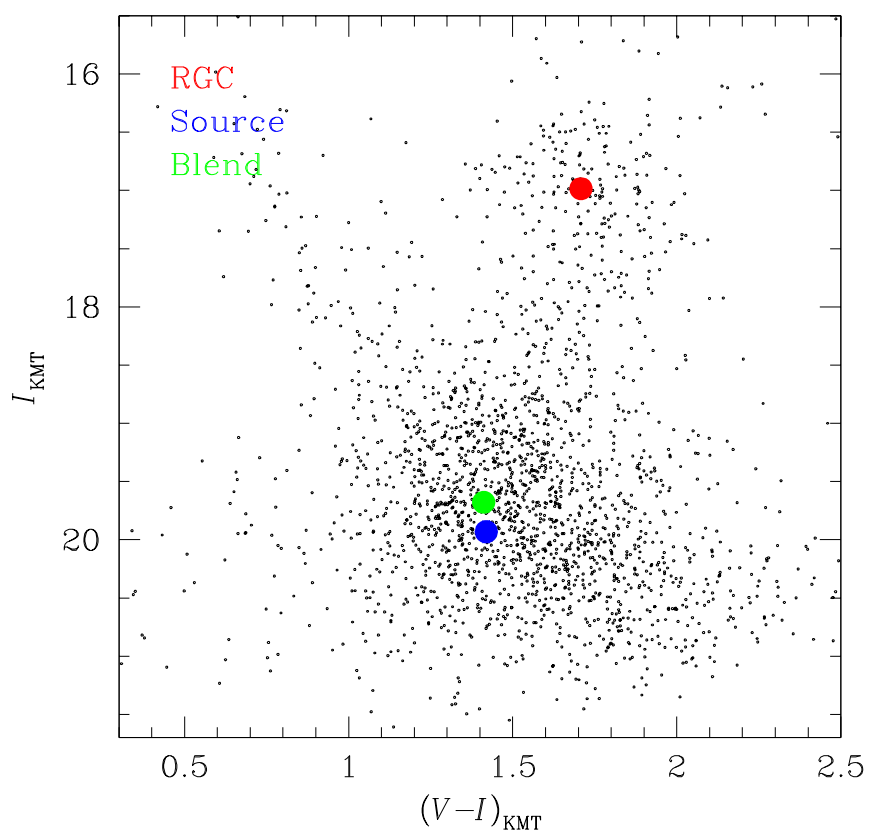

Figure 17. Position of the source with respect to the centroid of red giant clump (RGC) in the instrumental color-magnitude diagram of stars around the source. Also marked is the position of the blend.

Table 7

Source, Blend, Einstein Radius, and Proper Motion

\begin{tabular}{lr}
\hline \hline Quantity & \multicolumn{1}{c}{ Value } \\
\hline$(V-I)_{0}$ & $0.77 \pm 0.01$ \\
$I_{0}$ & $17.33 \pm 0.01$ \\
$I_{\mathrm{b}}$ & $19.04 \pm 0.01$ \\
$\theta_{*}(\mu$ as) & $1.98 \pm 0.08$ \\
$\theta_{\mathrm{E}}(\mathrm{mas})$ & $0.09 \pm 0.01$ \\
$\mu{\left.\text { (mas } \mathrm{yr}^{-1}\right)}^{-1}$ & $2.81 \pm 0.22$ \\
\hline
\end{tabular}

Note. The value $I_{\mathrm{b}}$ presents the $I$-band magnitude of the blend.

Chabrier (2003) for stars and that of Gould (2000) for stellar remnants. For the physical and dynamical distributions, we adopt the Han \& Gould $(2003,1995)$ models, respectively. For more details on these models, see Section 5 of Han et al. (2018). Based on these priors, we conduct a Monte Carlo simulation and produce $2 \times 10^{6}$ microlensing events. We then construct the distributions $M$ and $D_{\mathrm{L}}$ for events that have timescales and angular Einstein radii within the ranges of the measured $t_{\mathrm{E}}$ and $\theta_{\mathrm{E}}$. We estimate $M$ and $D_{\mathrm{L}}$ as the median values of the distributions, and their lower and upper limits are estimated as the 16th and 84th percentiles of the distributions, respectively.

In the Bayesian analysis, we also impose a constraint on the lens brightness such that the lens cannot be brighter than the measured blend brightness of $I_{\mathrm{b}} \sim 19.0$. The lens brightness is computed based on the mass, distance, and extinction. We assume that the extinction linearly increases with distance until it becomes $A_{I} \sim 1.68$ at $D_{\mathrm{S}}$, which is the measured value of the extinction toward the field. We find that this constraint has little effect on the lens mass and distance distributions. This is because lenses, in most cases, are much fainter than the blend. For the same reason, the result would not change with the choice of different extinction model along the line of sight. 

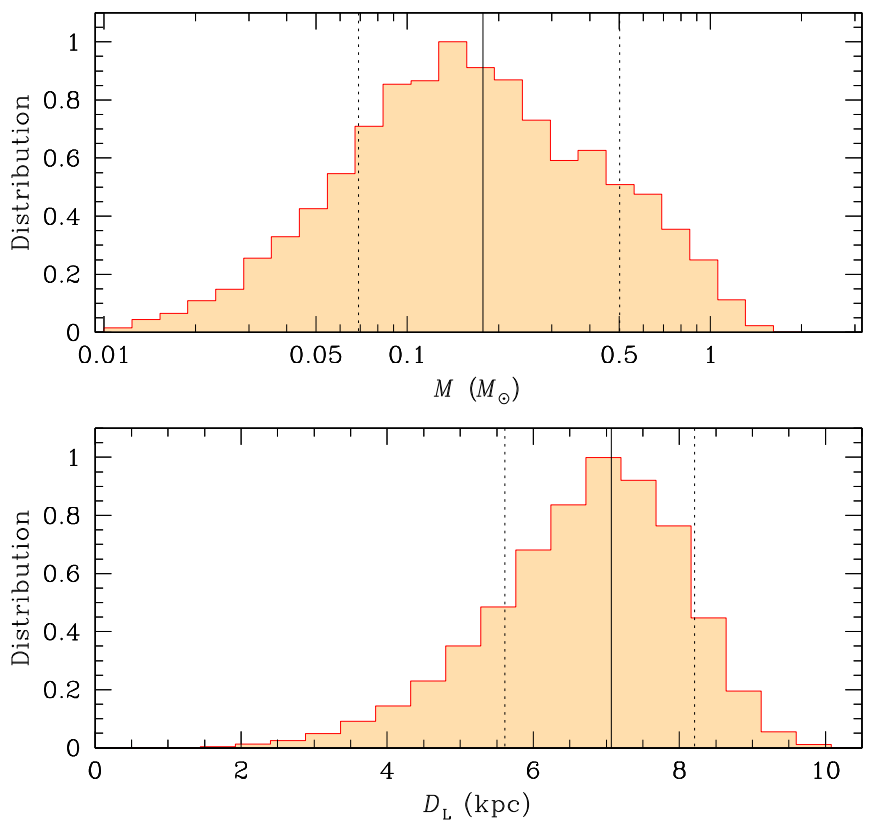

Figure 18. Distributions of the lens mass (upper panel) and distance (lower panel) estimated by Bayesian analysis. Solid vertical line for each distribution indicates the median value, and the two dotted vertical lines represent the $1 \sigma$ range of the estimated value, i.e., the 16th and 84th percentiles of the distribution.

In Figure 18, we present the distributions of the lens mass and the distance obtained from the Bayesian analysis. In Table 8, we list the estimated values of the individual lens components, $M_{1}, M_{2}$, and $M_{3}$, the distance to the lens, $D_{\mathrm{L}}$, and the projected separations of the planets from the host, $a_{\perp, 2}$ and $a_{\perp, 3}$. It is found that the lens is a multiple-planet system composed of two giant planets with masses

$$
M_{2}=1.8_{-1.1}^{+3.4} M_{\mathrm{J}}
$$

and

$$
M_{3}=2.8_{-1.7}^{+5.1} M_{\mathrm{J}}
$$

around a host star with a mass

$$
M_{1}=0.18_{-0.10}^{+0.33} M_{\odot} .
$$

The distance to the lens is estimated as

$$
D_{\mathrm{L}}=7.1_{-1.5}^{+1.1} \mathrm{kpc} \text {. }
$$

The estimated distance indicates that the lens is the farthest system among the known multiplanetary systems. We note that the previous multiplanetary system with the farthest distance is OGLE-2012-BLG-0026L, which is located $\sim 4.0 \pm 0.3 \mathrm{kpc}$ away (Han et al. 2013; Beaulieu et al. 2016). The projected separations of the planets from the host are

$$
a_{\perp, 2}=1.8_{-1.5}^{+2.1}\left(0.8_{-0.6}^{+0.9}\right) \text { au }
$$

and

$$
a_{\perp, 3}=0.8_{-0.6}^{+0.9} \text { au. }
$$

We note that the value of $a_{\perp, 2}$ in the parenthesis of Equation (11) is for the solution with $s_{2}<1.0$. The snow line of the planetary system is $a_{\mathrm{sl}} \sim 2.7 \mathrm{au}\left(M / M_{\odot}\right) \sim 0.5 \mathrm{au}$, and thus both planets are located beyond the snow line of the host,
Table 8

Physical Lens Parameters

\begin{tabular}{lc}
\hline \hline Parameter & Value \\
\hline$M_{1}\left(M_{\odot}\right)$ & $0.18_{-0.10}^{+0.33}$ \\
$M_{2}\left(M_{\mathrm{J}}\right)$ & $1.8_{-1.1}^{+3.4}$ \\
$M_{3}\left(M_{\mathrm{J}}\right)$ & $2.8_{-1.7}^{+5.1}$ \\
$D_{\mathrm{L}}(\mathrm{kpc})$ & $7.1_{-1.5}^{+1.1}$ \\
$a_{\perp, 2}(\mathrm{au})$ & $1.8_{-1.5}^{+2.1}\left(0.8_{-0.6}^{+0.9}\right)$ \\
$a_{\perp, 3}(\mathrm{au})$ & $0.8_{-0.6}^{+0.9}$ \\
\hline
\end{tabular}

Note. The $a_{\perp, 2}$ value in parenthesis is for the solution with $s_{2}>1.0$.

similar to the other cases of multiplanetary systems found by microlensing.

\section{Discussion and Conclusion}

We investigated the microlensing event OGLE-2018-BLG1011, for which the light curve exhibited an anomaly around the peak. We found that it was not possible to reasonably explain the anomaly with the binary-lens or binary-source interpretations, and its description required the introduction of an additional lens component. The 3L1S modeling resulted in three sets of solutions, in which one set of solutions indicated that the lens was a planetary system in a binary, while the other two sets of solutions implied that the lens was a multiplanetary system. By investigating the fits of the individual models to the detailed structure of the lensing light curve, we found that the multipleplanet solutions with planet-to-host mass ratios $\sim 9.5 \times 10^{-3}$ and $15 \times 10^{-3}$ were favored over the other solutions. From the Bayesian analysis for the best-fit solution, it was found that the lens is a multiple planetary system composed of giant planets with masses $\sim 1.8 M_{\mathrm{J}}$ and $\sim 2.8 M_{\mathrm{J}}$ orbiting a bulge star with a mass $\sim 0.18 M_{\odot}$ located at a distance of $\sim 7.1 \mathrm{kpc}$. The projected separations of the planets from the host were $a_{\perp, 2} \sim 1.8 \mathrm{au}$ (or $\sim 0.8 \mathrm{au}$ ) and $a_{\perp, 3} \sim 0.8 \mathrm{au}$, where the values of $a_{\perp, 2}$ denoted with and without parentheses were the separations corresponding to the two degenerate solutions with close and wide separations. Therefore, both planets were located beyond the snow line of the host, similar to the other four multiplanetary systems previously found by microlensing.

Work by C.H. was supported by the grant (2017R1A4A10151 78) of National Research Foundation of Korea. Work by A.G. was supported by US NSF grant AST-1516842. A.G. received support from the European Research Council under the European Union's Seventh Framework Programme (FP 7) ERC grant Agreement No. [321035]. The MOA project is supported by JSPS KAKENHI grant No. JSPS24253004, JSPS26247023, JSPS23340064, JSPS15H00781, and JP16H06287. Y.M. acknowledges support in the form of grant JP14002006. D.P.B., A.B., and C.R. were supported by NASA through grant NASA-80NSSC18K0274. The work by C.R. was supported by an appointment to the NASA Postdoctoral Program at the Goddard Space Flight Center, administered by USRA through a contract with NASA. NJR is a Royal Society of New Zealand Rutherford Discovery Fellow. The OGLE project has received funding from the National Science Centre, Poland, grant MAESTRO 2014/14/A/ST9/00121 to AU. This research has made use of the KMTNet system operated by the Korea Astronomy and Space Science Institute (KASI) and the data were obtained at three host sites of CTIO in Chile, SAAO in South 
Africa, and SSO in Australia. This research uses data obtained through the Telescope Access Program (TAP), which has been funded by the National Astronomical Observatories of China, the Chinese Academy of Sciences, and the Special Fund for Astronomy from the Ministry of Finance. W.Z., W.T., and S.M. acknowledge support by the National Science Foundation of China (Grant 11821303 and 11761131004). Work by W.Z., and P.F. was supported by Canada-France-Hawaii Telescope (CFHT). UKIRT is currently owned by the University of Hawaii (UH) and operated by the UH Institute for Astronomy; operations are enabled through the cooperation of the East Asian Observatory. The collection of the 2018 data reported here was supported by NASA grant NNG16PJ32C and JPL proposal \#18-NUP2018-0016. We also acknowledge the high-speed internet service (KREONET) provided by the Korea Institute of Science and Technology Information (KISTI).

\section{ORCID iDs}

Cheongho Han (D) https://orcid.org/0000-0002-2641-9964 David P. Bennett (1) https://orcid.org/0000-0001-8043-8413 Yossi Shvartzvald (iD https://orcid.org/0000-0003-1525-5041 Valerio Bozza (1) https://orcid.org/0000-0003-4590-0136 Sun-Ju Chung (1) https://orcid.org/0000-0001-6285-4528 Kyu-Ha Hwang (i) https://orcid.org/0000-0002-9241-4117 Yoon-Hyun Ryu (iD https://orcid.org/0000-0001-9823-2907 Jan Skowron (iD https://orcid.org/0000-0002-2335-1730 Paweł Pietrukowicz (i) https://orcid.org/0000-0002-2339-5899 Krzysztof Ulaczyk (i) https://orcid.org/0000-0001-6364-408X Patryk Iwanek (i) https://orcid.org/0000-0002-6212-7221 Akihiko Fukui (i) https://orcid.org/0000-0002-4909-5763 Yoshitaka Itow (ib https://orcid.org/0000-0002-8198-1968 Naoki Koshimoto (iD https://orcid.org/0000-0003-2302-9562 Shota Miyazaki (iD https://orcid.org/0000-0001-9818-1513 Nicholas J. Rattenbury (iD https://orcid.org/0000-0001-5069-319X Daisuke Suzuki (iD https://orcid.org/0000-0002-5843-9433 Matthew T. Penny (i) https://orcid.org/0000-0001-7506-5640 B. Scott Gaudi (i) https://orcid.org/0000-0003-0395-9869 Calen B. Henderson (iD https://orcid.org/0000-0001-8877-9060 Savannah Jacklin (iD https://orcid.org/0000-0001-9504-8258 Keivan G. Stassun (iD https://orcid.org/0000-0002-3481-9052

\section{References}

Alard, C., \& Lupton, R. H. 1998, ApJ, 503, 325

Albrow, M., Horne, K., Bramich, D. M., et al. 2009, MNRAS, 397, 2099 Albrow, M. D., Beaulieu, J.-P., Caldwell, J. A. R., et al. 2000, ApJ, 534, 894 Beaulieu, J.-P., Bennett, D. P., Batista, V., et al. 2016, ApJ, 824, 83 Bennett, D. P. 2010, ApJ, 716, 1408

Bennett, D. P., Rhie, S. H., Becker, A. C., et al. 1999, Natur, 402, 57 Bennett, D. P., Rhie, S. H., Nikolaev, S., et al. 2010, ApJ, 713, 837 Bennett, D. P., Rhie, S. H., Udalski, A., et al. 2016, AJ, 152, 125 Bensby, T., Adén, D., Meléndez, J., et al. 2011, A\&A, 533, 134
Bessell, M. S., \& Brett, J. M. 1988, PASP, 100, 1134

Bond, I. A., Abe, F., Dodd, R. J., et al. 2001, MNRAS, 327, 868 Bozza, V., Shvartzvald, Y., Udalski, A., et al. 2016, ApJ, 820, 79 Breger, M., Stich, J., Garrido, R., et al. 1993, A\&A, 271, 482 Chabrier, G. 2003, ApJL, 586, L133

Choi, J.-Y., Shin, I.-G., Han, C., et al. 2012, ApJ, 756, 48

Cleeves, L. I. 2016, ApJL, 816, L21

Daněk, K., \& Heyrovský, D. 2015, ApJ, 806, 99

Daněk, K., \& Heyrovský, D. 2019, ApJ, 880, 72

Dominik, M. 1998, A\&A, 329, 36

Dominik, M. 1999, A\&A, 349, 108

Dominik, M., Bachelet, E., Bozza, V., et al. 2019, MNRAS, 484, 5608

Gaia Collaboration, , et al. 2018, A\&A, 616, 1

Gaudi, B. S. 1998, ApJ, 506, 533

Gaudi, B. S., Bennett, D. P., Udalski, A., et al. 2008, Sci, 319, 927

Gaudi, B. S., \& Han, C. 2004, ApJ, 611, 528

Gaudi, B. S., Naber, R. M., \& Sackett, P. D. 1998, ApJL, 502, L33

Gould, A. 1992, ApJ, 392, 442

Gould, A. 2000, ApJ, 535, 928

Gould, A. 2014a, JKAS, 47, 215

Gould, A. 2014b, JKAS, 47, 153

Gould, A., Dong, S., Gaudi, B. S., et al. 2010, ApJ, 720, 1073

Gould, A., Udalski, A., Shin, I.-G., et al. 2014, Sci, 345, 46

Griest, K., \& Safazadeh, N. 1998, ApJ, 500, 37

Han, C. 2005, ApJ, 629, 1102

Han, C., Bennett, D. P., Udalski, A., \& Jung, Y. K. 2016, ApJ, 825, 8

Han, C., Bond, I. A., Gould, A., et al. 2018, AJ, 156, 226

Han, C., \& Gaudi, B. S. 2008, ApJ, 689, 53

Han, C., \& Gould, A. 1995, ApJ, 447, 53

Han, C., \& Gould, A. 2003, ApJ, 592, 172

Han, C., Udalski, A., Choi, J.-Y., et al. 2013, ApJL, 762, L28

Han, C., Udalski, A., Gould, A., et al. 2017, AJ, 154, 223

Hwang, K.-H., Choi, J.-Y., Bond, I. A., et al. 2013, ApJ, 778, 55

Ioka, K., Nishi, R., \& Kan-Ya, Y. 1999, PThPh, 102, 98

Jung, Y. K., Han, C., Gould, A., \& Maoz, D. 2013, ApJL, 768, L7

Jung, Y. K., Udalski, A., Yee, J. C., et al. 2017, AJ, 153, 129

Kervella, P., Thévenin, F., Di Folco, E., \& Ségransan, D. 2004, A\&A, 426, 29

Kim, D.-J., Kim, H.-W., Hwang, K.-H., et al. 2018, AJ, 155, 76

Kim, S.-L., Lee, C.-U., Park, B.-G., et al. 2016, JKAS, 49, 37

Lenz, P., \& Breger, M. 2005, CoAst, 146, 53

Lin, D. N. C., Bodenheimer, P., \& Richardson, D. C. 1996, Natur, 380, 606

Madsen, S., \& Zhu, W. 2019, ApJL, 878, L29

Mizuno, H. 1980, PThPh, 64, 544

Murray, N., Hansen, B., Holman, M., \& Tremaine, S. 1998, Sci, 279, 69

Nataf, D. M., Gould, A., Fouqué, P., et al. 2013, ApJ, 769, 88

Park, H., Han, C., Gould, A., et al. 2014, ApJ, 787, 71

Poleski, R., Skowron, J., Udalski, A., et al. 2014, ApJ, 795, 42

Pollack, J. B., Hubickyj, O., Bodenheimer, P., et al. 1996, Icar, 124, 62

Ryu, Y.-H., Udalski, A., Yee, J. C., et al. 2019, arXiv:1905.08148

Shin, I.-G., Yee, J. C., Gould, A., et al. 2019, arXiv:1902.10945

Shvartzvald, Y., Bryden, G., Gould, A., et al. 2017, AJ, 153, 61 Stevenson, D. J. 1982, P\&SS, 30, 755

Sumi, T., Abe, F., Bond, I. A., et al. 2003, ApJ, 591, 20

Suzuki, D., Bennett, D. P., Sumi, T., et al. 2016, ApJ, 833, 145

Suzuki, D., Bennett, D. P., Udalski, A., et al. 2018, AJ, 155, 263

Udalski, A., Jung, Y. K., Han, C., et al. 2015a, ApJ, 812, 47

Udalski, A., Szymański, M. K., \& Szymański, G. 2015b, AcA, 65, 1

Ward, W. R. 1997, Icar, 126, 261

Woźniak, P. R. 2000, AcA, 50, 421

Yee, J. C., Shvartzvald, Y., Gal-Yam, A., et al. 2012, ApJ, 755, 102

Yoo, J., DePoy, D. L., Gal-Yam, A., et al. 2004, ApJ, 603, 139

Zang, W., Penny, M., Zhu, W., et al. 2018, PASP, 130, 104401 